\title{
WELL-POSEDNESS OF THE NONLINEAR SCHRÖDINGER EQUATION ON THE HALF-PLANE
}

\author{
A. ALEXANDROU HIMONAS* \& DIONYSSIOS MANTZAVINOS
}

\begin{abstract}
The initial-boundary value problem (IBVP) for the nonlinear Schrödinger (NLS) equation on the half-plane with nonzero boundary data is studied by advancing a novel approach recently developed for the well-posedness of the cubic NLS on the half-line, which takes advantage of the solution formula produced by the unified transform of Fokas for the associated linear IBVP. For initial data in Sobolev spaces on the half-plane and boundary data in Bourgain spaces arising naturally when the linear IBVP is solved with zero initial data, the present work provides a local well-posedness result for NLS initial-boundary value problems in higher dimensions.
\end{abstract}

\section{Introduction AND Results}

We consider the following initial-boundary value problem (IBVP) for the two-dimensional nonlinear Schrödinger (NLS) equation on the half-plane:

$$
\begin{array}{ll}
i u_{t}+u_{x_{1} x_{1}}+u_{x_{2} x_{2}} \pm|u|^{p-1} u=0, & \left(x_{1}, x_{2}, t\right) \in \mathbb{R} \times \mathbb{R}^{+} \times(0, T), \\
u\left(x_{1}, x_{2}, 0\right)=u_{0}\left(x_{1}, x_{2}\right), & \left(x_{1}, x_{2}\right) \in \mathbb{R} \times \overline{\mathbb{R}^{+}}, \\
u\left(x_{1}, 0, t\right)=g_{0}\left(x_{1}, t\right), & \left(x_{1}, t\right) \in \mathbb{R} \times[0, T],
\end{array}
$$

where $p=3,5,7, \ldots$ and $T<1$, and prove its well-posedness for initial data $u_{0}\left(x_{1}, x_{2}\right)$ in the Sobolev space $H^{s}\left(\mathbb{R}_{x_{1}} \times \mathbb{R}_{x_{2}}^{+}\right), 1<s \leqslant \frac{3}{2}$, and boundary data $g_{0}\left(x_{1}, t\right)$ in a Bourgain space that arises naturally when the associated linear IBVP is solved with a zero initial datum. This result is proven by advancing into two dimensions a novel approach recently introduced for the wellposedness of IBVPs on the half-line [FHM1, FHM2, HM].

Remarkably, the Bourgain space associated with the one-dimensional NLS initial value problem (IVP) on the line emerges spontaneously in our work as the natural space for the boundary data. Furthermore, the regularity in the boundary variables $x_{1}, t$ of the solution of the linear Schrödinger IVP on the plane is described by the aforementioned Bourgain space, thereby extending into higher dimensions the result of [KPV1] on the time regularity of linear Schrödinger IVP on the line. In this respect, the Bourgain space from the one-dimensional NLS on the line comes forth as the optimal choice for the boundary data space of the two-dimensional NLS on the half-plane.

The NLS equation $i u_{t}+\Delta u \pm|u|^{p-1} u=0$ is a universal model, arising in multiple areas of mathematical physics such as nonlinear optics [Ta, AG], water waves [Z, HO, P, CSS], plasmas [WW], and Bose-Einstein condensates [PS, CP, KSS, CH]. As such, it has been studied extensively, from many points of view, and in a variety of different contexts. In one dimension, Zakharov and Shabat showed that the cubic case $p=3$ is a completely integrable system [ZS] (see also $[\mathrm{AKNS}])$. Thus, under the assumption of sufficient smoothness and decay, they were able to study

Date: October 4, 2018. *Corresponding author: himonas.1@nd.edu.

2010 Mathematics Subject Classification. Primary: 35Q55, 35G16, 35 G31.

Key words and phrases. 2D nonlinear Schrödinger equation, initial-boundary value problem, well-posedness, Sobolev spaces, Bourgain spaces, unified transform, Fokas method, linear space-time estimates, Laplace transform. 
the associated IVP on the line by analyzing the Lax pair of the equation via the inverse scattering transform. For rough data, the NLS IVP has been studied in great detail via harmonic analysis techniques. In particular, Tsutsumi [T2] proved global well-posedness in $L^{2}\left(\mathbb{R}^{n}\right)$ in the subcritical case $1<p<1+\frac{4}{n}$. Cazenave and Weissler [CW1] extended this result to the critical case $p=1+\frac{4}{n}$ and later to all $p>1$ for data in $H^{s}\left(\mathbb{R}^{n}\right), s>0$, using Besov spaces [CW2]. Other relevant works include Ginibre and Velo [GV1, GV2], Kato [K1, K2], and Constantin and Saut [CS]. The periodic case turned out to be more challenging and required the 1993 breakthrough of Bourgain [B1], who proved well-posedness for data in $H^{s}(\mathbb{T}), s \geqslant 0$, by introducing the celebrated $X^{s, b}$ spaces (with $b=\frac{1}{2}$ ). Further results on well-posedness and ill-posedness in one and higher dimensions for the periodic and the non-periodic IVP are available in [B2, B3, B4, KPV2, CKSTT, KV, D] and in numerous other works in the literature.

Contrary to the IVP, progress towards the rigorous analysis of IBVPs for NLS and other nonlinear evolution equations is rather limited. This can be largely attributed to the absence of the Fourier transform and underlying theory in the case of domains with a boundary. Recall, in particular, that the procedure for establishing local well-posedness of a nonlinear problem via a contraction mapping argument is initiated by defining an iteration map through the solution of the associated forced linear problem. In the case of the IVP, this task is straightforward thanks to the Fourier transform and Duhamel's principle. For IBVPs, however, a proper spatial transform is not available and hence a significant challenge is already present at the first step of the analysis. This difficulty is reflected in two independent approaches that were introduced in the early 2000s for showing well-posedness of the Korteweg-de Vries $(\mathrm{KdV})$ equation on the half-line, namely the works of Colliander and Kenig [CK] (in fact, this work is concerned with the generalized KdV equation) and of Bona, Sun and Zhang [BSZ1]. The first approach, which was later improved further for KdV and also adapted for NLS on the half-line by Holmer [H1, H2], is based on expressing the relevant forced linear IBVP as a chain of IVPs, thus allowing one to take advantage of the powerful Fourier analysis machinery but, at the same time, signifying a departure from the IBVP framework. The second approach, which has also been employed for NLS on the half-line [BSZ2], relies on solving the forced linear IBVP via a Laplace transform in the temporal variable, in contrast with the spatial (Fourier) transform used in the case of the IVP.

A novel approach was recently introduced for proving well-posedness of IBVPs for nonlinear evolution equations. This approach, which has already been implemented for the NLS, KdV and "good" Boussinesq equations on the half-line [FHM1, FHM2, HM], overcomes the lack of a proper spatial transform in the IBVP setting by exploiting the linear solution formulae produced via the unified transform, also known as the Fokas method [F1, F2]. Fokas' unified transform can be employed for solving linear evolution equations of arbitrary spatial order, supplemented with any type of admissible boundary data (including non-separable ones) and formulated in any number of spatial dimensions. In this light, taking also into account that no classical spatial transform exists for IBVPs involving linear evolution equations of order higher than two, the unified transform can be regarded as the analogue of the Fourier transform in the case of linear IBVPs. As such, it comes forth as the natural way of defining the iteration map to be used for showing well-posedness of nonlinear IBVPs via contraction mapping.

The essence of the new approach lies in the analysis of the pure linear IBVP, which corresponds to the case of zero initial datum and zero forcing. Indeed, the correct space for the boundary datum of the nonlinear problem is discovered in the process of estimating the unified transform solution formula of the pure linear problem. This step makes crucial use of the boundedness of the Laplace 
transform in $L^{2}$. Furthermore, it reveals the regularity of the solution of the linear IVP with respect to the boundary variable(s). In one dimension, this leads to the IVP time estimates previously obtained in [KPV1] (see also Theorem 4 in [FHM1] and Theorem 3.2); in higher dimensions, it provides the extension of these estimates, as given in Theorems 3.1 and 3.3.

Before stating our results precisely, we define the function spaces needed. The half-plane Sobolev space $H^{s}\left(\mathbb{R} \times \mathbb{R}^{+}\right)$for the initial data is defined as a restriction of the Sobolev space $H^{s}\left(\mathbb{R}^{2}\right)$ by

$$
H^{s}\left(\mathbb{R} \times \mathbb{R}^{+}\right)=\left\{f \in L^{2}\left(\mathbb{R} \times \mathbb{R}^{+}\right):\|f\|_{H^{s}\left(\mathbb{R} \times \mathbb{R}^{+}\right)} \doteq \inf _{\left.F\right|_{\mathbb{R} \times \mathbb{R}^{+}}=f}\|F\|_{H^{s}\left(\mathbb{R}^{2}\right)}<\infty\right\} .
$$

Furthermore, the space $B_{T}^{s}$ for the boundary data is defined as

$$
B_{T}^{s}=\left\{g \in L^{2}\left(\mathbb{R}_{x_{1}} \times[0, T]\right):\|g\|_{B_{T}^{s}} \doteq\|g\|_{H_{x_{1}}^{0} H_{t}^{\frac{2 s+1}{4}}}+\|g\|_{H_{x_{1}}^{s} H_{t}^{\frac{1}{4}}}<\infty\right\},
$$

where the two components of the norm $\|\cdot\|_{B_{T}^{s}}$ are given by

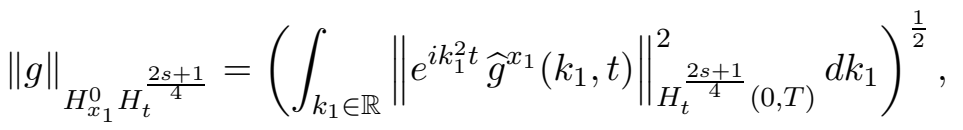

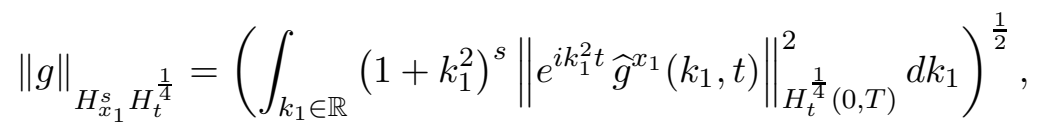

with $\widehat{g}^{x_{1}}\left(k_{1}, t\right)$ denoting the Fourier transform of $g\left(x_{1}, t\right)$ with respect to $x_{1}$, i.e.

$$
\widehat{g}^{x_{1}}\left(k_{1}, t\right)=\int_{x_{1} \in \mathbb{R}} e^{-i k_{1} x_{1}} g\left(x_{1}, t\right) d x_{1} .
$$

In fact, as shown in Section 2, a straightforward manipulation of the global-in-time counterparts of the norms (1.2) reveals that for $s \geqslant-\frac{1}{2}$ the space $B_{T}^{s}$ can be regarded as a restriction on $\mathbb{R}_{x_{1}} \times[0, T]$ of the space

$$
B^{s}=X^{0, \frac{2 s+1}{4}} \cap X^{s, \frac{1}{4}}, \quad\|g\|_{B^{s}} \doteq\|g\|_{X^{0, \frac{2 s+1}{4}}}+\|g\|_{X^{s, \frac{1}{4}}},
$$

where $X^{s, b}$ is the usual Bourgain space associated with the NLS IVP on the line, i.e.

$X^{s, b}=\left\{g \in L^{2}\left(\mathbb{R}_{x_{1}} \times \mathbb{R}_{t}\right):\|g\|_{X^{s, b}} \doteq\left(\int_{k_{1} \in \mathbb{R}} \int_{\tau \in \mathbb{R}}\left(1+k_{1}^{2}\right)^{s}\left(1+\left|\tau+k_{1}^{2}\right|\right)^{2 b}\left|\widehat{g}\left(k_{1}, \tau\right)\right|^{2} d \tau d k_{1}\right)^{\frac{1}{2}}<\infty\right\}$ with $\widehat{g}\left(k_{1}, \tau\right)$ here denoting the spatiotemporal Fourier transform of $g\left(x_{1}, t\right)$, that is

$$
\widehat{g}\left(k_{1}, \tau\right)=\int_{x_{1} \in \mathbb{R}} \int_{t \in \mathbb{R}} e^{-i k_{1} x_{1}-i \tau t} g\left(x_{1}, t\right) d t d x_{1} .
$$

We shall show that the NLS IBVP (1.1) is locally well-posed in the sense of Hadamard, namely that it possesses a unique solution which depends continuously on the prescribed initial and boundary data. We note that for $s>1$ the data must satisfy the compatibility condition

$$
u_{0}\left(x_{1}, 0\right)=g_{0}\left(x_{1}, 0\right) \quad \forall x_{1} \in \mathbb{R} .
$$

The precise statement of our main result is as follows.

Theorem 1.1 (Well-posedness of NLS on the half-plane). Suppose $1<s \leqslant \frac{3}{2}$. For initial data $u_{0} \in H^{s}\left(\mathbb{R}_{x_{1}} \times \mathbb{R}_{x_{2}}^{+}\right)$and boundary data $g_{0} \in B_{T}^{s}$ satisfying the compatibility condition (1.5), IBVP (1.1) for $N L S$ on the half-plane has a unique solution $u \in C\left(\left[0, T^{*}\right] ; H^{s}\left(\mathbb{R}_{x_{1}} \times \mathbb{R}_{x_{2}}^{+}\right)\right)$, which admits the estimate

$$
\sup _{t \in\left[0, T^{*}\right]}\|u(t)\|_{H^{s}\left(\mathbb{R}_{x_{1}} \times \mathbb{R}_{x_{2}}^{+}\right)} \leqslant 2 c_{s}\left\|\left(u_{0}, g_{0}\right)\right\|_{D}, \quad c_{s}=c(s)>0
$$


where $\left\|\left(u_{0}, g_{0}\right)\right\|_{D}=\left\|u_{0}\right\|_{H^{s}\left(\mathbb{R}_{x_{1}} \times \mathbb{R}_{x_{2}}^{+}\right)}+\left\|g_{0}\right\|_{B_{T}^{s}}$ and the lifespan $T^{*}$ is given by

$$
T^{*}=\min \left\{T, c_{s, p}\left\|\left(u_{0}, g_{0}\right)\right\|_{D}^{-2(p-1)}\right\}, \quad c_{s, p}=c(s, p)>0 .
$$

Moreover, the data-to-solution map $\left\{u_{0}, g_{0}\right\} \mapsto u$ is locally Lipschitz continuous.

We note that the vast majority of results in the literature on the Hadamard well-posedness of IBVPs for NLS in higher than one spatial dimensions refer to the case of zero (homogeneous) boundary data; see, for example, Brezis and Gallouet [BG], Y. Tsutsumi [T1], M. Tsutsumi [TM1, TM2], and Burq, Gérard and Tzvetkov [BGT1, BGT2]. Indeed, to the best of our knowledge, the only well-posedness results available for the non-homogeneous NLS IBVP (1.1) are those in the recent preprints by Audiard [AU] and Ran, Sun and Zhang [RSZ]. We emphasize, however, that the results of the present work are established via an entirely different method, namely by advancing into two spatial dimensions the novel approach which was recently introduced for one-dimensional IBVPs [FHM1, FHM2, HM] and which relies on Fokas' unified transform solution formulae. Taking into account the wide range of applicability of the unified transform, the approach developed in our work could be further adapted and generalized for showing well-posedness of IBVPs involving other well-known evolution equations in two as well as in higher dimensions.

Theorem 1.1 will be established via a contraction mapping argument. Hence, a key role in the analysis will be played by the forced linear analogue of the nonlinear problem (1.1), which reads

$$
\begin{array}{ll}
i u_{t}+u_{x_{1} x_{1}}+u_{x_{2} x_{2}}=f \in C\left([0, T] ; H^{s}\left(\mathbb{R}_{x_{1}} \times \mathbb{R}_{x_{2}}^{+}\right)\right), & \left(x_{1}, x_{2}, t\right) \in \mathbb{R} \times \mathbb{R}^{+} \times(0, T), \\
u\left(x_{1}, x_{2}, 0\right)=u_{0}\left(x_{1}, x_{2}\right) \in H^{s}\left(\mathbb{R}_{x_{1}} \times \mathbb{R}_{x_{2}}^{+}\right), & \left(x_{1}, x_{2}\right) \in \mathbb{R} \times \overline{\mathbb{R}^{+}}, \\
u\left(x_{1}, 0, t\right)=g_{0}\left(x_{1}, t\right) \in B_{T}^{s}, & \left(x_{1}, t\right) \in \mathbb{R} \times[0, T] .
\end{array}
$$

The forced linear IBVP (1.6) will be estimated by taking advantage of the following explicit solution formula obtained via the unified transform of Fokas:

$$
\begin{aligned}
u\left(x_{1}, x_{2}, t\right)= & S\left[u_{0}, g_{0} ; f\right]\left(x_{1}, x_{2}, t\right) \\
= & \frac{1}{(2 \pi)^{2}} \int_{k_{1} \in \mathbb{R}} \int_{k_{2} \in \mathbb{R}} e^{i k_{1} x_{1}+i k_{2} x_{2}-i\left(k_{1}^{2}+k_{2}^{2}\right) t} \widehat{u}_{0}\left(k_{1}, k_{2}\right) d k_{2} d k_{1} \\
& -\frac{1}{(2 \pi)^{2}} \int_{k_{1} \in \mathbb{R}} \int_{k_{2} \in \partial D} e^{i k_{1} x_{1}+i k_{2} x_{2}-i\left(k_{1}^{2}+k_{2}^{2}\right) t} \widehat{u}_{0}\left(k_{1},-k_{2}\right) d k_{2} d k_{1} \\
& -\frac{i}{(2 \pi)^{2}} \int_{k_{1} \in \mathbb{R}} \int_{k_{2} \in \mathbb{R}} e^{i k_{1} x_{1}+i k_{2} x_{2}-i\left(k_{1}^{2}+k_{2}^{2}\right) t} \int_{t^{\prime}=0}^{t} e^{i\left(k_{1}^{2}+k_{2}^{2}\right) t^{\prime}} \widehat{f}\left(k_{1}, k_{2}, t^{\prime}\right) d t^{\prime} d k_{2} d k_{1} \\
& +\frac{i}{(2 \pi)^{2}} \int_{k_{1} \in \mathbb{R}} \int_{k_{2} \in \partial D} e^{i k_{1} x_{1}+i k_{2} x_{2}-i\left(k_{1}^{2}+k_{2}^{2}\right) t} \int_{t^{\prime}=0}^{t} e^{i\left(k_{1}^{2}+k_{2}^{2}\right) t^{\prime}} \widehat{f}\left(k_{1},-k_{2}, t^{\prime}\right) d t^{\prime} d k_{2} d k_{1} \\
& +\frac{1}{(2 \pi)^{2}} \int_{k_{1} \in \mathbb{R}} \int_{k_{2} \in \partial D} e^{i k_{1} x_{1}+i k_{2} x_{2}-i\left(k_{1}^{2}+k_{2}^{2}\right) t} 2 k_{2}{\widehat{g_{0}}}^{T}\left(k_{1},-k_{1}^{2}-k_{2}^{2}\right) d k_{2} d k_{1} .
\end{aligned}
$$

In the above formula, $\widehat{u}_{0}$ stands for the half-plane Fourier transform of the initial datum $u_{0}$, i.e.

$$
\widehat{u}_{0}\left(k_{1}, k_{2}\right)=\int_{x_{1} \in \mathbb{R}} \int_{x_{2} \in \mathbb{R}^{+}} e^{-i k_{1} x_{1}-i k_{2} x_{2}} u_{0}\left(x_{1}, x_{2}\right) d x_{2} d x_{1},
$$

which is well-defined for $k_{1} \in \mathbb{R}$ and $k_{2} \in \overline{\mathbb{C}^{-}}=\left\{k_{2} \in \mathbb{C}: \operatorname{Im}\left(k_{2}\right) \leqslant 0\right\}$ due to the fact that $x_{2} \geqslant 0$. Moreover, ${\widehat{g_{0}}}^{T}$ denotes the spatiotemporal transform

$$
{\widehat{g_{0}}}^{T}\left(k_{1}, \tau\right)=\int_{x_{1} \in \mathbb{R}} \int_{t=0}^{T} e^{-i k_{1} x_{1}-i \tau t} g_{0}\left(x_{1}, t\right) d t d x_{1},
$$


which makes sense for $k_{1} \in \mathbb{R}$ and $\tau \in \mathbb{C}$ since integration in $t$ takes place over a bounded interval. Finally, the contour of integration $\partial D$ is the positively oriented boundary of the first quadrant $D$ of the complex $k_{2}$-plane, as shown in Figure 1.1. A concise derivation of formula (1.7), whose homogeneous version was first derived in [F3], is provided in the appendix.

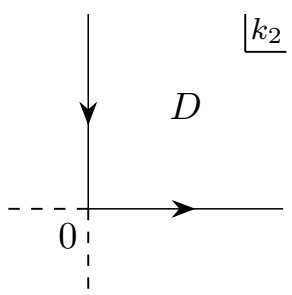

Figure 1.1. The region $D$ and its positively oriented boundary $\partial D$.

Starting from the unified transform formula (1.7), we shall establish the following result, which, apart from being instrumental in the proof of Theorem 1.1 for the well-posedness of the nonlinear problem (1.1), is interesting in its own right in regard to the forced linear problem (1.6).

Theorem 1.2 (Forced linear Schrödinger on the half-plane). Suppose that $1<s \leqslant \frac{3}{2}$. Then, the unified transform formula (1.7) defines a solution $u=S\left[u_{0}, g_{0} ; f\right]$ to the forced linear Schrödinger IBVP (1.6) supplemented with the compatibility condition (1.5), which satisfies the estimate

$$
\sup _{t \in[0, T]}\|u(t)\|_{H^{s}\left(\mathbb{R}_{x_{1}} \times \mathbb{R}_{x_{2}}^{+}\right)} \leqslant c_{s}\left(\left\|u_{0}\right\|_{H^{s}\left(\mathbb{R}_{x_{1}} \times \mathbb{R}_{x_{2}}^{+}\right)}+\left\|g_{0}\right\|_{B_{T}^{s}}+\sqrt{T} \sup _{t \in[0, T]}\|f(t)\|_{H^{s}\left(\mathbb{R}_{x_{1}} \times \mathbb{R}_{x_{2}}^{+}\right)}\right),
$$

where $c_{s}>0$ is a constant that depends only on $s$.

The literature on the IVP for the NLS equation in one and higher dimensions is extensive. Further results on the well-posedness, stability and blow-up behavior of NLS via hard analysis as well as integrability techniques can be found in [W, LPSS, GS, KPV3, M, CKS, DZ, SS, BGT3, $\mathrm{MR}, \mathrm{V}, \mathrm{KTV}, \mathrm{LP}, \mathrm{FH}, \mathrm{BM}]$ and the references therein. Moreover, for a thorough introduction to Fokas' unified transform method we refer the reader to the monograph [F2] and the review article [FS]. In particular, we note that the IBVP on the half-line and on the interval for the cubic NLS equation has been studied via the integrable nonlinear extension of the unified transform by Fokas and collaborators in [FIS] and [FI] respectively. Other works via similar techniques include [FP, F4, LF1, LF2, MF].

Structure of the paper. In Section 2, employing the Fokas unified transform formula (1.7) and exploiting the boundedness of the Laplace transform in $L^{2}$, we estimate the solution of the pure linear Schrödinger IBVP, i.e. of problem (1.6) with zero initial datum and zero forcing. The resulting estimate is key to our analysis, as it reveals the boundary data space $B_{T}^{s}$. In Section 3 , we show that the $x_{1} t$-regularity of the solution of both the homogeneous and the forced linear Schrödinger on the whole line is described by $B_{T}^{s}$. Then, in Section 4 , we combine the estimates of the previous sections to deduce Theorem 1.2 for the forced linear IBVP (1.6). This provides the central estimate needed for the proof of Theorem 1.1 on the well-posedness of NLS on the half-plane, which is carried out in Section 5 via a contraction mapping argument. Finally, a concise derivation of the unified transform solution formula (1.7) is provided in the appendix. 


\section{The Pure Linear IBVP}

The essence of the analysis of the forced linear IBVP (1.6) is captured in the simplest genuine such problem, namely an IBVP with zero initial datum and forcing but with a nonzero boundary datum (note that the case of zero boundary datum can be reduced to an IVP). In fact, this "model problem" can be simplified further by assuming a boundary datum with compact support in the $t$-variable. Thus, we are led to the following problem, which we identify as the pure linear IBVP:

$$
\begin{array}{ll}
i v_{t}+v_{x_{1} x_{1}}+v_{x_{2} x_{2}}=0, & \left(x_{1}, x_{2}, t\right) \in \mathbb{R} \times \mathbb{R}^{+} \times(0,2), \\
v\left(x_{1}, x_{2}, 0\right)=0, & \left(x_{1}, x_{2}\right) \in \mathbb{R} \times \overline{\mathbb{R}^{+}}, \\
v\left(x_{1}, 0, t\right)=g\left(x_{1}, t\right), & \left(x_{1}, t\right) \in \mathbb{R} \times[0,2], \\
\operatorname{supp}(g) \subset \mathbb{R}_{x_{1}} \times(0,2) . &
\end{array}
$$

As we shall see below, the estimation of the solution of the pure linear IBVP (2.1) in the usual Sobolev space $H^{s}\left(\mathbb{R} \times \mathbb{R}^{+}\right)$reveals the "exotic" Bourgain-type space $B^{s}$ and, in turn, the restriction space $B_{T}^{s}$ for the boundary datum of the NLS IBVP (1.1). We note that, contrary to the half-line, where placing the initial datum in $H^{s}\left(\mathbb{R}^{+}\right.$) automatically requires a Sobolev boundary datum (see Theorem 5 in [FHM1]), in the case of the half-plane it is not a priori clear what is the appropriate space for the boundary datum. In this respect, the pure linear IBVP holds a central role in the analysis of both the linear and the nonlinear problem. Another source of motivation for the boundary data space $B_{T}^{s}$ is the linear Schrödinger IVP (see Theorem 3.1), in a less direct way, however.

The general Fokas unified transform formula (1.7) yields the solution of IBVP (2.1) as

$$
\begin{aligned}
v\left(x_{1}, x_{2}, t\right) & =S[0, g ; 0]\left(x_{1}, x_{2}, t\right) \\
& =\frac{1}{(2 \pi)^{2}} \int_{k_{1} \in \mathbb{R}} \int_{k_{2} \in \partial D} e^{i k_{1} x_{1}+i k_{2} x_{2}-i\left(k_{1}^{2}+k_{2}^{2}\right) t} 2 k_{2} \widehat{g}\left(k_{1},-k_{1}^{2}-k_{2}^{2}\right) d k_{2} d k_{1},
\end{aligned}
$$

where the contour $\partial D$ is depicted in Figure 1.1. Importantly, note that thanks to the support assumption (2.1d) the boundary datum $g$ appears in the above formula through its two-dimensional Fourier transform (1.4) instead of the "truncated" transform (1.9).

Theorem 2.1 (Pure IBVP estimate). The solution $v=S[0, g ; 0]$ of the pure linear IBVP (2.1) given by formula (2.2) admits the estimate

$$
\sup _{t \in[0,2]}\|v(t)\|_{H^{s}\left(\mathbb{R}_{x_{1}} \times \mathbb{R}_{x_{2}}^{+}\right)} \leqslant c_{s}\|g\|_{B^{s}}, \quad s \geqslant 0,
$$

Remark 2.1. In fact, one can also prove the following estimate:

$$
\sup _{x_{2} \in \overline{\mathbb{R}^{+}}}\left\|v\left(x_{2}\right)\right\|_{B^{s}} \leqslant c_{s}\|g\|_{B^{s}}, \quad s \in \mathbb{R},
$$

which allows one to carry out the contraction for the nonlinear IBVP in the space $C\left(\left[0, T^{*}\right] ; H^{s}(\mathbb{R})\right) \cap$ $C\left(\mathbb{R}_{x_{2}}^{+} ; B_{T^{*}}^{s}\right)$ instead of the Hadamard space of Theorem 1.1.

Proof of Theorem 2.1. We decompose formula (2.2) in two parts as $v=v_{1}+v_{2}$ where

$$
v_{1}\left(x_{1}, x_{2}, t\right)=\frac{1}{(2 \pi)^{2}} \int_{k_{1} \in \mathbb{R}} \int_{k_{2}=0}^{\infty} e^{i k_{1} x_{1}-k_{2} x_{2}-i\left(k_{1}^{2}-k_{2}^{2}\right) t} 2 k_{2} \widehat{g}\left(k_{1},-k_{1}^{2}+k_{2}^{2}\right) d k_{2} d k_{1}
$$

corresponds to the imaginary axis portion of $\partial D$ and

$$
v_{2}\left(x_{1}, x_{2}, t\right)=\frac{1}{(2 \pi)^{2}} \int_{k_{1} \in \mathbb{R}} \int_{k_{2}=0}^{\infty} e^{i k_{1} x_{1}+i k_{2} x_{2}-i\left(k_{1}^{2}+k_{2}^{2}\right) t} 2 k_{2} \widehat{g}\left(k_{1},-k_{1}^{2}-k_{2}^{2}\right) d k_{2} d k_{1}
$$


corresponds to the real axis part of $\partial D$. The estimation of $v_{2}$ is easier, while that of $v_{1}$ is more challenging and relies crucially on the boundedness of the Laplace transform in $L^{2}$.

Estimation along the real axis. Let $V_{2}\left(x_{1}, x_{2}, t\right)$ be a global-in-space function defined via the twodimensional Fourier transform

$$
\widehat{V}_{2}\left(k_{1}, k_{2}, t\right)= \begin{cases}e^{-i\left(k_{1}^{2}+k_{2}^{2}\right) t} 2 k_{2} \widehat{g}\left(k_{1},-k_{1}^{2}-k_{2}^{2}\right), & k_{2}>0, \\ 0, & k_{2} \leqslant 0,\end{cases}
$$

so that $\left.V_{2}\right|_{x_{2} \in \mathbb{R}^{+}}=v_{2}$. In turn, we have

$$
\left\|v_{2}(t)\right\|_{H^{s}\left(\mathbb{R}_{x_{1}} \times \mathbb{R}_{x_{2}}^{+}\right)}^{2} \leqslant\left\|V_{2}(t)\right\|_{H^{s}\left(\mathbb{R}^{2}\right)}^{2}=\int_{k_{1} \in \mathbb{R}} \int_{k_{2} \in \mathbb{R}}\left(1+k_{1}^{2}+k_{2}^{2}\right)^{s}\left|\widehat{V}_{2}\left(k_{1}, k_{2}, t\right)\right|^{2} d k_{2} d k_{1}
$$

and hence for $s \geqslant 0$ we find

$$
\begin{aligned}
\left\|v_{2}(t)\right\|_{H^{s}\left(\mathbb{R}_{x_{1}} \times \mathbb{R}_{x_{2}}^{+}\right)}^{2} & \int_{k_{1} \in \mathbb{R}} \int_{k_{2}=0}^{\infty}\left(1+k_{1}^{2}\right)^{s} k_{2}^{2}\left|\widehat{g}\left(k_{1},-k_{1}^{2}-k_{2}^{2}\right)\right|^{2} d k_{2} d k_{1} \\
& +\int_{k_{1} \in \mathbb{R}} \int_{k_{2}=0}^{\infty}\left(k_{2}^{2}\right)^{s+1}\left|\widehat{g}\left(k_{1},-k_{1}^{2}-k_{2}^{2}\right)\right|^{2} d k_{2} d k_{1}
\end{aligned}
$$

Making the change of variable $k_{2}=\sqrt{-\tau}$ and using the property $\widehat{\varphi}^{t}(\tau-a)={\widehat{e^{i a t} \varphi(t)}}^{t}(\tau)$, the above inequality becomes

$$
\begin{aligned}
& \left\|v_{2}(t)\right\|_{H^{s}\left(\mathbb{R}_{x_{1}} \times \mathbb{R}_{x_{2}}^{+}\right)}^{2} \lesssim \int_{k_{1} \in \mathbb{R}} \int_{\tau=-\infty}^{0}\left(1+k_{1}^{2}\right)^{s}|\tau|^{\frac{1}{2}}\left|{\overline{e^{i k_{1}^{2} t} \widehat{g}^{x_{1}}\left(k_{1}, t\right)}}^{t}(\tau)\right|^{2} d \tau d k_{1} \\
& +\int_{k_{1} \in \mathbb{R}} \int_{\tau=-\infty}^{0}|\tau|^{s+\frac{1}{2}}\left|{\widehat{e^{i k_{1}^{2} t} \widehat{g}^{x_{1}}\left(k_{1}, t\right)}}^{t}(\tau)\right|^{2} d \tau d k_{1} .
\end{aligned}
$$

Thus, since $s \geqslant 0>-\frac{1}{2}$, we have

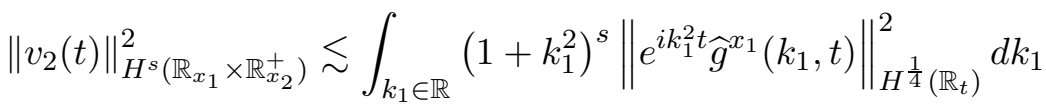

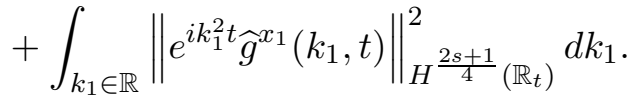

The two terms on the right-hand side of the above estimate are the global-in-time counterparts of the components $(1.2)$ of the $B_{T}^{s}$-norm. In this connection, we note that

$$
\begin{aligned}
\|g\|_{X^{0, \frac{2 s+1}{4}}}^{2} & =\int_{k_{1} \in \mathbb{R}} \int_{\tau \in \mathbb{R}}\left(1+\left|\tau+k_{1}^{2}\right|\right)^{2 \cdot \frac{2 s+1}{4}}\left|\widehat{g}\left(k_{1}, \tau\right)\right|^{2} d \tau d k_{1} \\
& \simeq \int_{k_{1} \in \mathbb{R}} \int_{\tau \in \mathbb{R}}\left(1+\tau^{2}\right)^{\frac{2 s+1}{4}}\left|\widehat{g}\left(k_{1}, \tau-k_{1}^{2}\right)\right|^{2} d \tau d k_{1}=(2.7 \mathrm{~b})
\end{aligned}
$$

and, similarly,

$$
\|g\|_{X^{s, \frac{1}{4}}}^{2} \simeq \int_{k_{1} \in \mathbb{R}}\left(1+k_{1}^{2}\right)^{s} \int_{\tau \in \mathbb{R}}\left(1+\tau^{2}\right)^{\frac{1}{4}}\left|\widehat{g}\left(k_{1}, \tau-k_{1}^{2}\right)\right|^{2} d \tau d k_{1}=(2.7 \mathrm{a}) .
$$

Thus, by the definition of the $B^{s}$-norm, estimate (2.7) is equivalent to

$$
\left\|v_{2}(t)\right\|_{H^{s}\left(\mathbb{R}_{x_{1}} \times \mathbb{R}_{x_{2}}^{+}\right)} \lesssim\|g\|_{B^{s}} .
$$


Estimation along the imaginary axis. We employ the physical space equivalent Sobolev norm

$$
\left\|v_{1}(t)\right\|_{H^{s}\left(\mathbb{R}_{x_{1}} \times \mathbb{R}_{x_{2}}^{+}\right)}^{2}=\sum_{|\alpha| \leqslant\lfloor s\rfloor}\left\|\partial_{x}^{\alpha} v_{1}(t)\right\|_{L^{2}\left(\mathbb{R}_{x_{1}} \times \mathbb{R}_{x_{2}}^{+}\right)}^{2}+\sum_{|\alpha|=\lfloor s\rfloor}\left\|\partial_{x}^{\alpha} v_{1}(t)\right\|_{\beta}^{2}, \quad s \geqslant 0
$$

where $x=\left(x_{1}, x_{2}\right)$ and $\partial_{x}^{\alpha}=\partial_{x_{1}}^{\alpha_{1}} \partial_{x_{2}}^{\alpha_{2}}$ with $|\alpha|=\alpha_{1}+\alpha_{2}$, and for $\beta=s-\lfloor s\rfloor \in(0,1)$ we define

$$
\begin{aligned}
\left\|\partial_{x}^{\alpha} v_{1}(t)\right\|_{\beta}^{2} & =\iint_{x \in \mathbb{R} \times \mathbb{R}^{+}} \iint_{y \in \mathbb{R} \times \mathbb{R}^{+}} \frac{\left|\partial_{x}^{\alpha} v_{1}(x, t)-\partial_{x}^{\alpha} v_{1}(y, t)\right|^{2}}{|x-y|^{2(1+\beta)}} d y d x \\
& \simeq \int_{x \in \mathbb{R} \times \mathbb{R}^{+}} \int_{z \in \mathbb{R} \times \mathbb{R}^{+}} \frac{\left|\partial_{x}^{\alpha} v_{1}(x+z, t)-\partial_{x}^{\alpha} v_{1}(x, t)\right|^{2}}{|z|^{2(1+\beta)}} d z d x .
\end{aligned}
$$

Estimation of the integer part. We begin with the estimation of $\left\|\partial_{x}^{\alpha} v_{1}(t)\right\|_{L^{2}\left(\mathbb{R}_{x_{1}} \times \mathbb{R}_{x_{2}}^{+}\right)}$for all $|\alpha| \in \mathbb{N}_{0}$ with $|\alpha| \leqslant\lfloor s\rfloor$. Differentiating formula (2.5), we have

$$
\partial_{x}^{\alpha} v_{1}\left(x_{1}, x_{2}, t\right) \simeq \int_{k_{2}=0}^{\infty} e^{-k_{2} x_{2}} G\left(k_{2}, x_{1}, t\right) d k_{2},
$$

where

$$
G\left(k_{2}, x_{1}, t\right)=\int_{k_{1} \in \mathbb{R}} e^{i k_{1} x_{1}-i\left(k_{1}^{2}-k_{2}^{2}\right) t} k_{1}^{\alpha_{1}} k_{2}^{\alpha_{2}} k_{2} \widehat{g}\left(k_{1},-k_{1}^{2}+k_{2}^{2}\right) d k_{1} .
$$

Thus,

$$
\left\|\partial_{x}^{\alpha} v_{1}(t)\right\|_{L^{2}\left(\mathbb{R}_{x_{1}} \times \mathbb{R}_{x_{2}}^{+}\right)}^{2} \simeq \int_{x_{1} \in \mathbb{R}}\left\|\mathcal{L}\left\{G\left(k_{2}, x_{1}, t\right)\right\}\left(x_{2}\right)\right\|_{L^{2}\left(\mathbb{R}_{x_{2}}^{+}\right)}^{2} d x_{1},
$$

where $\mathcal{L}\{G\}$ denotes the Laplace transform of $G$ with respect to $k_{2}$, i.e.

$$
\mathcal{L}\left\{G\left(k_{2}, x_{1}, t\right)\right\}\left(x_{2}\right)=\int_{k_{2}=0}^{\infty} e^{-k_{2} x_{2}} G\left(k_{2}, x_{1}, t\right) d k_{2} .
$$

Lemma 2.1 ( $L^{2}$-boundedness of the Laplace transform). The map

$$
\mathcal{L}: \phi \mapsto \int_{k_{2}=0}^{\infty} e^{-k_{2} x_{2}} \phi\left(k_{2}\right) d k_{2}
$$

is bounded from $L_{k_{2}}^{2}(0, \infty)$ into $L_{x_{2}}^{2}(0, \infty)$ with

$$
\|\mathcal{L}\{\phi\}\|_{L_{x_{2}}^{2}(0, \infty)} \leqslant \sqrt{\pi}\|\phi\|_{L_{k_{2}}^{2}(0, \infty)} .
$$

A proof of Lemma 2.1 is available in [FHM1]. Using the relevant estimate for $\phi\left(k_{2}\right)=G\left(k_{2}, x_{1}, t\right)$, we obtain

$$
\begin{aligned}
\left\|\partial_{x}^{\alpha} v_{1}(t)\right\|_{L^{2}\left(\mathbb{R}_{x_{1}} \times \mathbb{R}_{x_{2}}^{+}\right)}^{2} & \lesssim \int_{x_{1} \in \mathbb{R}} \int_{k_{2}=0}^{\infty}\left|\int_{k_{1} \in \mathbb{R}} e^{i k_{1} x_{1}-i\left(k_{1}^{2}-k_{2}^{2}\right) t} k_{1}^{\alpha_{1}} k_{2}^{\alpha_{2}} k_{2} \widehat{g}\left(k_{1},-k_{1}^{2}+k_{2}^{2}\right) d k_{1}\right|^{2} d k_{2} d x_{1} \\
& \simeq \int_{k_{2}=0}^{\infty} \int_{k_{1} \in \mathbb{R}}\left(k_{1}^{2}\right)^{\alpha_{1}}\left(k_{2}^{2}\right)^{\alpha_{2}} k_{2}^{2}\left|\widehat{g}\left(k_{1},-k_{1}^{2}+k_{2}^{2}\right)\right|^{2} d k_{1} d k_{2}
\end{aligned}
$$

after also applying Parseval-Plancherel in $x_{1}$ and $k_{1}$. 
Therefore,

$$
\begin{aligned}
\sum_{|\alpha| \leqslant\lfloor s\rfloor}\left\|\partial_{x}^{\alpha} v_{1}(t)\right\|_{L^{2}\left(\mathbb{R}_{x_{1}} \times \mathbb{R}_{x_{2}}^{+}\right)}^{2} & \lesssim \sum_{|\alpha| \leqslant\lfloor s\rfloor} \int_{k_{2}=0}^{\infty} \int_{k_{1} \in \mathbb{R}}\left(k_{1}^{2}\right)^{\alpha_{1}}\left(k_{2}^{2}\right)^{\alpha_{2}} k_{2}^{2}\left|\widehat{g}\left(k_{1},-k_{1}^{2}+k_{2}^{2}\right)\right|^{2} d k_{1} d k_{2} \\
& \simeq \int_{k_{2}=0}^{\infty} \int_{k_{1} \in \mathbb{R}}\left(\sum_{|\alpha|=0}^{\lfloor s\rfloor}\left(k_{1}^{2}+k_{2}^{2}\right)^{|\alpha|}\right) k_{2}^{2}\left|\widehat{g}\left(k_{1},-k_{1}^{2}+k_{2}^{2}\right)\right|^{2} d k_{1} d k_{2} \\
& \lesssim \int_{k_{2}=0}^{\infty} \int_{k_{1} \in \mathbb{R}}\left(k_{1}^{2}+k_{2}^{2}\right)^{s} k_{2}^{2}\left|\widehat{g}\left(k_{1},-k_{1}^{2}+k_{2}^{2}\right)\right|^{2} d k_{1} d k_{2}
\end{aligned}
$$

Then, since $\left(k_{1}^{2}+k_{2}^{2}\right)^{s} \lesssim\left(1+k_{1}^{2}\right)^{s}+\left(k_{2}^{2}\right)^{s}$ for $s \geqslant 0$, we infer

$$
\begin{aligned}
\sum_{|\alpha| \leqslant\lfloor s\rfloor}\left\|\partial_{x}^{\alpha} v_{1}(t)\right\|_{L^{2}\left(\mathbb{R}_{x_{1}} \times \mathbb{R}_{x_{2}}^{+}\right)}^{2} \lesssim & \int_{k_{2}=0}^{\infty} \int_{k_{1} \in \mathbb{R}}\left(1+k_{1}^{2}\right)^{s} k_{2}^{2}\left|\widehat{g}\left(k_{1},-k_{1}^{2}+k_{2}^{2}\right)\right|^{2} d k_{1} d k_{2} \\
& +\int_{k_{2}=0}^{\infty} \int_{k_{1} \in \mathbb{R}}\left(k_{2}^{2}\right)^{s} k_{2}^{2}\left|\widehat{g}\left(k_{1},-k_{1}^{2}+k_{2}^{2}\right)\right|^{2} d k_{1} d k_{2} .
\end{aligned}
$$

Finally, making the change of variable $k_{2}=\sqrt{\tau}$, we conclude in view of (2.8) that

$$
\begin{aligned}
\sum_{|\alpha| \leqslant\lfloor s\rfloor}\left\|\partial_{x}^{\alpha} v_{1}(t)\right\|_{L^{2}\left(\mathbb{R}_{x_{1}} \times \mathbb{R}_{x_{2}}^{+}\right) \lesssim}^{2} & \int_{\tau=0}^{\infty} \int_{k_{1} \in \mathbb{R}}\left(1+k_{1}^{2}\right)^{s} \sqrt{\tau}\left|\widehat{g}\left(k_{1}, \tau-k_{1}^{2}\right)\right|^{2} d k_{1} d \tau \\
& +\int_{\tau=0}^{\infty} \int_{k_{1} \in \mathbb{R}} \tau^{s+\frac{1}{2}}\left|\widehat{g}\left(k_{1}, \tau-k_{1}^{2}\right)\right|^{2} d k_{1} d \tau \\
\leqslant & \|g\|_{X^{s, \frac{1}{4}}}^{2}+\|g\|_{X^{0, \frac{2 s+1}{4}}}^{2} \leqslant\|g\|_{B^{s}}^{2} .
\end{aligned}
$$

Estimation of the fractional part. We proceed to the estimation of $\left\|\partial_{x}^{\alpha} v_{1}(t)\right\|_{\beta}$ where now $|\alpha|=\alpha_{1}+\alpha_{2}=\lfloor s\rfloor \in \mathbb{N}_{0}$. By the definition (2.11) of the fractional norm and Parseval-Plancherel in $x_{1}$ and $k_{1}$, we have

$$
\begin{aligned}
\left\|\partial_{x}^{\alpha} v_{1}(t)\right\|_{\beta}^{2} \simeq & \int_{z_{1} \in \mathbb{R}} \int_{x_{2}=0}^{\infty} \int_{z_{2}=0}^{\infty} \frac{1}{|z|^{2(1+\beta)}} \int_{x_{1} \in \mathbb{R}} \mid \int_{k_{1} \in \mathbb{R}} e^{i k_{1} x_{1}}\left[k_{1}^{\alpha_{1}} e^{-i k_{1}^{2} t}\right. \\
& \left.\cdot \int_{k_{2}=0}^{\infty} e^{-k_{2} x_{2}+i k_{2}^{2} t}\left(e^{i k_{1} z_{1}-k_{2} z_{2}}-1\right) k_{2}^{\alpha_{2}} k_{2} \widehat{g}\left(k_{1},-k_{1}^{2}+k_{2}^{2}\right) d k_{2}\right]\left.d k_{1}\right|^{2} d x_{1} d z_{2} d x_{2} d z_{1} \\
\simeq & \int_{k_{1} \in \mathbb{R}} k_{1}^{2 \alpha_{1}} \int_{z_{1} \in \mathbb{R}} \int_{z_{2}=0}^{\infty} \frac{1}{|z|^{2(1+\beta)}} \\
& \cdot \int_{x_{2}=0}^{\infty}\left|\int_{k_{2}=0}^{\infty} e^{-k_{2} x_{2}+i k_{2}^{2} t}\left(e^{i k_{1} z_{1}-k_{2} z_{2}}-1\right) k_{2}^{\alpha_{2}} k_{2} \widehat{g}\left(k_{1},-k_{1}^{2}+k_{2}^{2}\right) d k_{2}\right|^{2} d x_{2} d z_{2} d z_{1} d k_{1} .
\end{aligned}
$$

Thus, using the Laplace transform Lemma 2.1 in $x_{2}$ and $k_{2}$, we obtain

$$
\left\|\partial_{x}^{\alpha} v_{1}(t)\right\|_{\beta}^{2} \lesssim \int_{k_{1} \in \mathbb{R}} k_{1}^{2 \alpha_{1}} \int_{k_{2}=0}^{\infty} I\left(k_{1}, k_{2}, \beta\right) k_{2}^{2 \alpha_{2}} k_{2}^{2}\left|\widehat{g}\left(k_{1},-k_{1}^{2}+k_{2}^{2}\right)\right|^{2} d k_{2} d k_{1}
$$


where

$$
\begin{aligned}
I\left(k_{1}, k_{2}, \beta\right) & =\int_{z_{1} \in \mathbb{R}} \int_{z_{2}=0}^{\infty} \frac{\left|e^{i k_{1} z_{1}-k_{2} z_{2}}-1\right|^{2}}{\left(z_{1}^{2}+z_{2}^{2}\right)^{1+\beta}} d z_{2} d z_{1} \\
& =\frac{1}{\left|k_{1}\right| k_{2}} \int_{\zeta_{1} \in \mathbb{R}} \int_{\zeta_{2}=0}^{\infty} \frac{\left|e^{i \zeta_{1}-\zeta_{2}}-1\right|^{2}}{\left(\frac{\zeta_{1}^{2}}{k_{1}^{2}}+\frac{\zeta_{2}^{2}}{k_{2}^{2}}\right)^{1+\beta}} d \zeta_{2} d \zeta_{1}
\end{aligned}
$$

Lemma 2.2. The integral I defined by (2.16) admits the bound

$$
I\left(k_{1}, k_{2}, \beta\right) \lesssim\left(k_{1}^{2}+k_{2}^{2}\right)^{\beta}, \quad \beta \in(0,1)
$$

Lemma 2.2 is proven after the end of the current proof. Combining estimate (2.17) with inequality (2.15), we deduce

$$
\left\|\partial_{x}^{\alpha} v_{1}(t)\right\|_{\beta}^{2} \lesssim \int_{k_{1} \in \mathbb{R}} k_{1}^{2 \alpha_{1}} \int_{k_{2}=0}^{\infty}\left(k_{1}^{2}+k_{2}^{2}\right)^{\beta} k_{2}^{2 \alpha_{2}} k_{2}^{2}\left|\widehat{g}\left(k_{1},-k_{1}^{2}+k_{2}^{2}\right)\right|^{2} d k_{2} d k_{1} .
$$

Therefore, recalling that $\alpha_{2}=|\alpha|-\alpha_{1}$, we obtain

$$
\begin{aligned}
\sum_{|\alpha|=\lfloor s\rfloor}\left\|\partial_{x}^{\alpha} v_{1}(t)\right\|_{\beta}^{2} & \lesssim \sum_{|\alpha|=\lfloor s\rfloor} \int_{k_{1} \in \mathbb{R}} \int_{k_{2}=0}^{\infty}\left(k_{1}^{2}+k_{2}^{2}\right)^{\beta} k_{1}^{2 \alpha_{1}} k_{2}^{2 \alpha_{2}} k_{2}^{2}\left|\widehat{g}\left(k_{1},-k_{1}^{2}+k_{2}^{2}\right)\right|^{2} d k_{2} d k_{1} \\
& \simeq \int_{k_{1} \in \mathbb{R}} \int_{k_{2}=0}^{\infty}\left(k_{1}^{2}+k_{2}^{2}\right)^{\beta}\left(k_{1}^{2}+k_{2}^{2}\right)^{\lfloor s\rfloor} k_{2}^{2}\left|\widehat{g}\left(k_{1},-k_{1}^{2}+k_{2}^{2}\right)\right|^{2} d k_{2} d k_{1} \\
& =\int_{k_{1} \in \mathbb{R}} \int_{k_{2}=0}^{\infty}\left(k_{1}^{2}+k_{2}^{2}\right)^{s} k_{2}^{2}\left|\widehat{g}\left(k_{1},-k_{1}^{2}+k_{2}^{2}\right)\right|^{2} d k_{2} d k_{1} .
\end{aligned}
$$

The right-hand side above is the same with that of (2.13). Hence, proceeding as before we conclude that

$$
\sum_{|\alpha|=\lfloor s\rfloor}\left\|\partial_{x}^{\alpha} v_{1}(t)\right\|_{\beta} \lesssim\|g\|_{B^{s}}, \quad s \geqslant 0 .
$$

The integer estimate (2.14) and the fractional estimate (2.18) combined with the definition (2.10) of the Sobolev norm yield

$$
\left\|v_{1}(t)\right\|_{H^{s}\left(\mathbb{R}_{x_{1}} \times \mathbb{R}_{x_{2}}^{+}\right)} \lesssim\|g\|_{B^{s}}, \quad s \geqslant 0 .
$$

This estimate together with estimate (2.9) for $v_{2}$ implies estimate (2.3) for the solution $v$ of the pure linear IBVP.

The proof of Theorem 2.1 is complete.

Proof of Lemma 2.2. Note that $I$ is even in $k_{1}$. Hence, without loss of generality, we estimate $I$ for $\left(k_{1}, k_{2}\right) \in \mathbb{R}^{+} \times \mathbb{R}^{+}$. There are two cases to consider: (i) $k_{2}=\lambda k_{1}, 0 \leqslant \lambda \leqslant 1$, and (ii) $k_{1}=\lambda k_{2}$, $0 \leqslant \lambda \leqslant 1$. In the first case, starting from (2.16) we have

$$
\begin{aligned}
I=I\left(k_{1}, \lambda k_{1}, \beta\right) & =\frac{\left(k_{1}^{2}\right)^{1+\beta}}{\lambda k_{1}^{2}} \int_{\zeta_{1} \in \mathbb{R}} \int_{\zeta_{2}=0}^{\infty} \frac{\left|e^{i \zeta_{1}-\zeta_{2}}-1\right|^{2}}{\left(\zeta_{1}^{2}+\frac{\zeta_{2}^{2}}{\lambda^{2}}\right)^{1+\beta}} d \zeta_{2} d \zeta_{1} \\
& =\left(k_{1}^{2}\right)^{\beta} \int_{\zeta_{1} \in \mathbb{R}} \int_{\zeta_{2}=0}^{\infty} \frac{\left|e^{i \zeta_{1}-\lambda \zeta_{2}}-1\right|^{2}}{\left(\zeta_{1}^{2}+\zeta_{2}^{2}\right)^{1+\beta}} d \zeta_{2} d \zeta_{1}=\left(k_{1}^{2}\right)^{\beta} J_{1}(\lambda, \beta),
\end{aligned}
$$

after letting $\zeta_{2} \mapsto \lambda \zeta_{2}$. Switching to polar coordinates $\zeta_{1}=r \cos \theta, \zeta_{2}=r \sin \theta$ yields

$$
J_{1}(\lambda, \beta)=\int_{\theta=0}^{\pi} \int_{r=0}^{\infty} \frac{\left|e^{(i \cos \theta-\lambda \sin \theta) r}-1\right|^{2}}{r^{1+2 \beta}} d r d \theta .
$$


For $r \ll 1$, we have $e^{(i \cos \theta-\lambda \sin \theta) r}=1+(i \cos \theta-\lambda \sin \theta) r+O\left(r^{2}\right)$ thus the integrand of $J_{1}$ becomes

$$
\frac{\left|e^{(i \cos \theta-\lambda \sin \theta) r}-1\right|^{2}}{r^{1+2 \beta}}=\frac{1}{r^{2 \beta-1}}|(i \cos \theta-\lambda \sin \theta)+O(r)|^{2},
$$

which is integrable at $r=0$ since $\beta<1$. Furthermore, for $r \gg 1$ the integrand of $J_{1}$ becomes

$$
\frac{\left|e^{(i \cos \theta-\lambda \sin \theta) r}-1\right|^{2}}{r^{1+2 \beta}} \leqslant \frac{(1+1)^{2}}{r^{1+2 \beta}},
$$

which is integrable at $r=\infty$ since $\beta>0$. Thus, $J_{1}(\lambda, \beta)=c_{\lambda, \beta}<\infty$ and hence

$$
I\left(k_{1}, \lambda k_{1}, \beta\right) \lesssim\left(k_{1}^{2}\right)^{\beta}, \quad 0 \leqslant \lambda \leqslant 1 .
$$

A similar argument shows that

$$
I\left(\lambda k_{2}, k_{2}, \beta\right) \lesssim\left(k_{2}^{2}\right)^{\beta}, \quad 0 \leqslant \lambda \leqslant 1
$$

Combining estimates (2.20) and (2.21) concludes the proof of Lemma 2.2.

\section{Linear IVP Estimates}

The pure linear IBVP (2.1) will be combined with appropriate linear IVPs to yield the forced linear IBVP (1.6) via the superposition principle. The details of this (de)composition are given in Section 4. Hence, Theorem 2.1 for the forced linear IBVP will be established by combining the estimate of Theorem 2.1 for the pure linear IBVP with suitable estimates for the aforementioned IVPs. These estimates are derived below.

Homogeneous linear IVP estimates. We begin with the linear Schrödinger IVP

$$
\begin{array}{ll}
i U_{t}+U_{x_{1} x_{1}}+U_{x_{2} x_{2}}=0, & \left(x_{1}, x_{2}, t\right) \in \mathbb{R} \times \mathbb{R} \times(0, T), \\
U\left(x_{1}, x_{2}, 0\right)=U_{0}\left(x_{1}, x_{2}\right) \in H^{s}\left(\mathbb{R}_{x_{1}} \times \mathbb{R}_{x_{2}}\right), & \left(x_{1}, x_{2}\right) \in \mathbb{R} \times \mathbb{R},
\end{array}
$$

whose solution is given by

$$
U\left(x_{1}, x_{2}, t\right)=S\left[U_{0} ; 0\right]\left(x_{1}, x_{2}, t\right)=\frac{1}{(2 \pi)^{2}} \int_{k_{1} \in \mathbb{R}} \int_{k_{2} \in \mathbb{R}} e^{i k_{1} x_{1}+i k_{2} x_{2}-i\left(k_{1}^{2}+k_{2}^{2}\right) t} \widehat{U}_{0}\left(k_{1}, k_{2}\right) d k_{2} d k_{1},
$$

where $\widehat{U}_{0}$ is the Fourier transform of $U_{0}$ on the plane defined by

$$
\widehat{U}_{0}\left(k_{1}, k_{2}\right)=\int_{x_{1} \in \mathbb{R}} \int_{x_{2} \in \mathbb{R}} e^{-i k_{1} x_{1}-i k_{2} x_{2}} U_{0}\left(x_{1}, x_{2}\right) d x_{2} d x_{1} .
$$

We shall discover below that regularity of the solution of IVP (3.1) in the variables $x_{1}$ and $t$ is described by the boundary data space $B_{T}^{s}$. This result can be regarded as the two-dimensional analogue of the time estimate of [KPV1], which states that the solution of the linear Schrödinger IVP on the line with initial data in $H^{s}\left(\mathbb{R}_{x}\right)$ belongs to $H^{\frac{2 s+1}{4}}(0, T)$ as a function of $t$. Hence, both in one and in two dimensions, the regularity of the linear IVP with respect to the boundary variables is described by the boundary data space of the associated Dirichlet IBVP. The precise statement of our result is the following.

Theorem 3.1 (Homogeneous IVP estimates). The solution $U=S\left[U_{0} ; 0\right]$ of the homogeneous linear IVP (3.1) given by formula (3.2) satisfies the estimates

$$
\begin{array}{ll}
\sup _{t \in[0, T]}\|U(t)\|_{H^{s}\left(\mathbb{R}_{x_{1}} \times \mathbb{R}_{x_{2}}\right)}=\left\|U_{0}\right\|_{H^{s}\left(\mathbb{R}_{x_{1}} \times \mathbb{R}_{x_{2}}\right)}, & s \in \mathbb{R}, \\
\sup _{x_{2} \in \mathbb{R}}\left\|U\left(x_{2}\right)\right\|_{B_{T}^{s}} \leqslant c_{s}\left\|U_{0}\right\|_{H^{s}\left(\mathbb{R}_{x_{1}} \times \mathbb{R}_{x_{2}}\right)}, & s \geqslant 0 .
\end{array}
$$


Remark 3.1. Recall that $B_{T}^{s}$ emerges naturally in the proof of estimate (2.3) as the boundary data space that allows for the solution of the pure linear IBVP to belong in $H^{s}\left(\mathbb{R} \times \mathbb{R}^{+}\right)$. Estimate (3.5) indicates an alternative, reverse path for discovering $B_{T}^{s}$, namely by investigating the $x_{1} t$-regularity of the solution of the linear Schrödinger IVP when the initial datum belongs in $H^{s}(\mathbb{R} \times \mathbb{R})$. Together, estimates (2.3) and (3.5) can be visualized as the upper and lower half of a closed loop from the data space to the solution space and back, and hence confirm that $B_{T}^{s}$ is indeed the correct boundary data space for NLS on the half-plane in the case of Sobolev initial data.

Proof of Theorem 3.1. The isometry relation (3.4) follows easily from formula (3.2) and the definition of the Sobolev norm. Concerning estimate (3.5), we note that the function

$$
Q\left(k_{1}, x_{2}, t\right)=e^{i k_{1}^{2} t} \widehat{U}^{x_{1}}\left(k_{1}, x_{2}, t\right)
$$

involved in the $B_{T}^{s}$-norm of $U$ satisfies for all $k_{1} \in \mathbb{R}$ the one-dimensional IVP

$$
\begin{array}{ll}
i Q_{t}+Q_{x_{2} x_{2}}=0, & \left(x_{2}, t\right) \in \mathbb{R} \times(0, T), \\
Q\left(k_{1}, x_{2}, 0\right)={\widehat{U_{0}}}^{x_{1}}\left(k_{1}, x_{2}\right), & x_{2} \in \mathbb{R} .
\end{array}
$$

Thus, we have the following estimate from Theorem 4 of [FHM1]:

$$
\left\|Q\left(k_{1}, x_{2}\right)\right\|_{H_{t}^{\frac{2 s+1}{4}}(0, T)} \lesssim\left\|\widehat{U}_{0}^{x_{1}}\left(k_{1}\right)\right\|_{H^{s}\left(\mathbb{R}_{x_{2}}\right)}, \quad s \geqslant-\frac{1}{2}, k_{1}, x_{2} \in \mathbb{R} .
$$

In turn, for all $x_{2} \in \mathbb{R}$ and $s \geqslant-\frac{1}{2}$ we infer

$$
\begin{aligned}
\left\|U\left(x_{2}\right)\right\|_{B_{T}^{s}}^{2} & \lesssim \int_{k_{1} \in \mathbb{R}}\left\|\widehat{U}_{0}^{x_{1}}\left(k_{1}, x_{2}\right)\right\|_{H^{s}\left(\mathbb{R}_{x_{2}}\right)}^{2} d k_{1}+\int_{k_{1} \in \mathbb{R}}\left(1+k_{1}^{2}\right)^{s}\left\|\widehat{U}_{0}^{x_{1}}\left(k_{1}, x_{2}\right)\right\|_{H^{0}\left(\mathbb{R}_{x_{2}}\right)}^{2} d k_{1} \\
& =\int_{k_{1} \in \mathbb{R}} \int_{k_{2} \in \mathbb{R}}\left[\left(1+k_{1}^{2}\right)^{s}+\left(1+k_{2}^{2}\right)^{s}\right]\left|\widehat{U}_{0}\left(k_{1}, k_{2}\right)\right|^{2} d k_{2} d k_{1},
\end{aligned}
$$

which implies estimate (3.5) upon restricting $s \geqslant 0$.

Forced linear IVP estimates. We continue with the estimation of the forced linear Schrödinger on the plane. First, we need a result for the one-dimensional forced IVP

$$
\begin{array}{ll}
i w_{t}+w_{x x}=f(x, t), & (x, t) \in \mathbb{R} \times \mathbb{R}, \\
w(x, 0)=0, & x \in \mathbb{R},
\end{array}
$$

whose solution is given by

$$
\begin{aligned}
w(x, t)=S[0 ; f](x, t) & =-\frac{i}{2 \pi} \int_{t^{\prime}=0}^{t} \int_{k \in \mathbb{R}} e^{i k x-i k^{2}\left(t-t^{\prime}\right)} \widehat{f}\left(k, t^{\prime}\right) d k d t^{\prime} \\
& =-i \int_{t^{\prime}=0}^{t} S\left[f\left(\cdot, t^{\prime}\right) ; 0\right]\left(x, t-t^{\prime}\right) d t^{\prime}
\end{aligned}
$$

where $\widehat{f}$ is the whole-line Fourier transform of $f$ defined by

$$
\widehat{f}(k, t)=\int_{x \in \mathbb{R}} e^{-i k x} f(x, t) d x
$$

and $S\left[f\left(x, t^{\prime}\right) ; 0\right]$ denotes the solution of the one-dimensional homogeneous IVP (3.6) with initial datum $f\left(x, t^{\prime}\right)$. 
Theorem 3.2 (Forced IVP time estimate in one dimension). For all $x \in \mathbb{R}$, the solution $w=S[0 ; f]$ of the one-dimensional forced linear IVP (3.8) given by formula (3.9) admits the bounds

$$
\begin{aligned}
\|w(x)\|_{H_{t}^{\frac{2 s+1}{4}}(0, T)}^{2} \lesssim & \int_{t=0}^{T}\|f(t)\|_{H^{s}\left(\mathbb{R}_{x}\right)}^{2} d t \\
& +\int_{t=0}^{T} \int_{z=0}^{T-t} \frac{1}{z^{\frac{3}{2}+s}}\left[\int_{t^{\prime}=t}^{t+z}\left\|f\left(t^{\prime}\right)\right\|_{H^{s}\left(\mathbb{R}_{x}\right)} d t^{\prime}\right]^{2} d z d t, \quad \frac{1}{2}<s<\frac{3}{2}, \\
\|w(x)\|_{H_{t}^{\frac{2 s+1}{4}}(0, T)}^{2} \lesssim \int_{t=0}^{T}\|f(t)\|_{H^{s}\left(\mathbb{R}_{x}\right)}^{2} d t, & s=0, \frac{3}{2} .
\end{aligned}
$$

Proof of Theorem 3.3. Let $m=\frac{2 s+1}{4}$ and note that $0 \leqslant m<1$ corresponds to $-\frac{1}{2} \leqslant s<\frac{3}{2}$. Hence, in this range of $s$ the physical space equivalent $H_{t}^{m}(0, T)$-norm reads

$$
\|w(x)\|_{H_{t}^{m}(0, T)}^{2}=\|w(x)\|_{L_{t}^{2}(0, T)}^{2}+\|w(x)\|_{m}^{2}, \quad 0 \leqslant m<1,
$$

where the fractional part of the norm is defined for $m \in(0,1)$ by

$$
\|w(x)\|_{m}^{2}=\int_{t=0}^{T} \int_{z=0}^{T-t} \frac{|w(x, t+z)-w(x, t)|^{2}}{z^{1+2 m}} d z d t .
$$

Starting from formula (3.9b) and using Minkowski's integral inequality and the homogeneous IVP time estimate (3.7), we find

$$
\|w(x)\|_{L_{t}^{2}(0, T)} \lesssim \int_{t^{\prime}=0}^{T}\left\|S\left[f\left(\cdot, t^{\prime}\right) ; 0\right]\left(x, t-t^{\prime}\right)\right\|_{L_{t}^{2}(0, T)} d t^{\prime} \lesssim \int_{t^{\prime}=0}^{T}\left\|f\left(t^{\prime}\right)\right\|_{H^{-\frac{1}{2}\left(\mathbb{R}_{x}\right)}} d t^{\prime} .
$$

Moreover, employing the Duhamel representation (3.9b), we have

$$
\begin{aligned}
\|w(x)\|_{m}^{2} \leqslant & \int_{t=0}^{T} \int_{z=0}^{T-t} \frac{1}{z^{1+2 m}}\left(\int_{t^{\prime}=0}^{T}\left|S\left[f\left(\cdot, t^{\prime}\right) ; 0\right]\left(x, t+z-t^{\prime}\right)-S\left[f\left(\cdot, t^{\prime}\right) ; 0\right]\left(x, t-t^{\prime}\right)\right| d t^{\prime}\right)^{2} d z d t \\
& +\int_{t=0}^{T} \int_{z=0}^{T-t} \frac{1}{z^{1+2 m}}\left|\int_{t^{\prime}=t}^{t+z} S\left[f\left(\cdot, t^{\prime}\right) ; 0\right]\left(x, t+z-t^{\prime}\right) d t^{\prime}\right|^{2} d z d t .
\end{aligned}
$$

The term (3.15a) can be estimated via Minkowski's integral inequality in the $t^{\prime}$ - and $z t$-integrals, followed by the homogeneous IVP time estimate (3.7). Eventually, we find

$$
(3.15 \mathrm{a}) \lesssim \int_{t=0}^{T}\|f(t)\|_{H^{s}\left(\mathbb{R}_{x}\right)}^{2} d t .
$$

For the term (3.15b), we treat the cases $m>\frac{1}{2}$ and $m<\frac{1}{2}$ separately. In the former case, using formula (3.9a) and applying Cauchy-Schwarz inequality in $k$, we have

$$
\begin{aligned}
(3.15 \mathrm{~b}) & \lesssim \int_{t=0}^{T} \int_{z=0}^{T-t} \frac{1}{z^{1+2 m}}\left(\int_{k \in \mathbb{R}} \int_{t^{\prime}=t}^{t+z}\left|\widehat{f}\left(k, t^{\prime}\right)\right| d t^{\prime} d k\right)^{2} d z d t \\
& \lesssim \int_{t=0}^{T} \int_{z=0}^{T-t} \frac{1}{z^{1+2 m}} \int_{k \in \mathbb{R}}\left(1+k^{2}\right)^{s}\left(\int_{t^{\prime}=t}^{t+z}\left|\widehat{f}\left(k, t^{\prime}\right)\right| d t^{\prime}\right)^{2} d k d z d t .
\end{aligned}
$$

where we have made crucial use of the fact that $m>\frac{1}{2}$ implies $s>\frac{1}{2}$ and hence $\int_{k \in \mathbb{R}} \frac{d k}{\left(1+k^{2}\right)^{s}}<\infty$. Thus, Minkowski's integral inequality in $t^{\prime}$ and $k$ yields

$$
(3.15 \mathrm{~b}) \lesssim \int_{t=0}^{T} \int_{z=0}^{T-t} \frac{1}{z^{1+2 m}}\left[\int_{t^{\prime}=t}^{t+z}\left\|f\left(t^{\prime}\right)\right\|_{H^{s}\left(\mathbb{R}_{x}\right)} d t^{\prime}\right]^{2} d z d t .
$$


Combining estimates (3.16) and (3.17), we deduce the estimate

$\|w(x)\|_{m}^{2} \lesssim \int_{t^{\prime}=0}^{T}\left\|f\left(t^{\prime}\right)\right\|_{H^{s}\left(\mathbb{R}_{x}\right)}^{2} d t^{\prime}+\int_{t=0}^{T} \int_{z=0}^{T-t} \frac{1}{z^{1+2 m}}\left[\int_{t^{\prime}=t}^{t+z}\left\|f\left(t^{\prime}\right)\right\|_{H^{s}\left(\mathbb{R}_{x}\right)} d t^{\prime}\right]^{2} d z d t, \quad \frac{1}{2}<m<1$, which, together with the $L^{2}$-estimate (3.14), implies the bound (3.24a).

For $m<\frac{1}{2}$, applying Cauchy-Schwarz inequality in $t^{\prime}$ and then interchanging the $t$ - and $z$ integrals, we have

$$
(3.15 \mathrm{~b}) \leqslant \int_{z=0}^{T} \frac{1}{z^{2 m}} \int_{t=0}^{T-z} \int_{t^{\prime}=t}^{t+z}\left|S\left[f\left(\cdot, t^{\prime}\right) ; 0\right]\left(x, t+z-t^{\prime}\right)\right|^{2} d t^{\prime} d t d z .
$$

Hence, letting $t \rightarrow t-z$ and augmenting the range of integration with respect to $t$ and $t^{\prime}$, we find

$$
\begin{aligned}
(3.15 \mathrm{~b}) & \leqslant \int_{z=0}^{T} \frac{1}{z^{2 m}} \int_{t=z}^{T} \int_{t^{\prime}=t-z}^{t}\left|S\left[f\left(\cdot, t^{\prime}\right) ; 0\right]\left(x, t-t^{\prime}\right)\right|^{2} d t^{\prime} d t d z \\
& \leqslant\left(\int_{z=0}^{T} \frac{1}{z^{2 m}} d z\right) \int_{t^{\prime}=0}^{T}\left\|S\left[f\left(\cdot, t^{\prime}\right) ; 0\right]\left(x, t-t^{\prime}\right)\right\|_{L_{t}^{2}(0, T)}^{2} d t^{\prime},
\end{aligned}
$$

so, integrating in $z$ (recall that $2 m<1$ ) and using the homogeneous IVP time estimate $(3.7)$, we obtain

$$
(3.15 \mathrm{~b}) \leqslant \frac{T^{1-2 m}}{1-2 m} \int_{t=0}^{T}\|f(t)\|_{H^{-\frac{1}{2}\left(\mathbb{R}_{x}\right)}}^{2} d t .
$$

Estimates (3.16) and (3.18) together imply

$$
\|w(x)\|_{m}^{2} \lesssim \int_{t=0}^{T}\|f(t)\|_{H^{s}\left(\mathbb{R}_{x}\right)}^{2} d t+\frac{T^{1-2 m}}{1-2 m} \int_{t=0}^{T}\|f(t)\|_{H^{-\frac{1}{2}\left(\mathbb{R}_{x}\right)}}^{2} d t, \quad 0<m<\frac{1}{2},
$$

thus, recalling also the $L^{2}$-estimate (3.14) and the fact that $T<1$, we deduce

$$
\|w(x)\|_{H_{t}^{m}(0, T)}^{2} \lesssim \frac{1}{1-2 m} \int_{t=0}^{T}\|f(t)\|_{H^{s}\left(\mathbb{R}_{x}\right)}^{2} d t, \quad 0 \leqslant m<\frac{1}{2},
$$

which is the bound (3.11b) for $s=0$.

Finally, in order to establish (3.11b) for $s=\frac{3}{2}$ (which corresponds to $m=1$ ) we need to estimate

$$
\|w(x)\|_{H_{t}^{1}(0, T)}^{2}=\|w(x)\|_{L_{t}^{2}(0, T)}^{2}+\left\|\partial_{t} w(x)\right\|_{L_{t}^{2}(0, T)}^{2} .
$$

The first $L^{2}$-norm above was estimated earlier — see (3.14). Furthermore, differentiating the Duhamel representation $(3.9 \mathrm{~b})$ with respect to $t$, we have

$$
\left\|\partial_{t} w(x)\right\|_{L_{t}^{2}(0, T)}^{2} \lesssim\|f(x)\|_{L_{t}^{2}(0, T)}^{2}+\left\|\int_{t^{\prime}=0}^{t} S\left[\partial_{x}^{2} f\left(\cdot, t^{\prime}\right) ; 0\right]\left(x, t-t^{\prime}\right) d t^{\prime}\right\|_{L_{t}^{2}(0, T)}^{2} .
$$

Since $s>\frac{1}{2}$, the Sobolev embedding theorem in $x$ implies

$$
\|f(x)\|_{L_{t}^{2}(0, T)}^{2} \leqslant \int_{t=0}^{T}\|f(t)\|_{L^{\infty}\left(\mathbb{R}_{x}\right)}^{2} d t \leqslant \int_{t=0}^{T}\|f(t)\|_{H^{s}\left(\mathbb{R}_{x}\right)}^{2} d t .
$$

Moreover, similarly to the derivation of (3.14), we have

$$
\left\|\int_{t^{\prime}=0}^{t} S\left[\partial_{x}^{2} f\left(t^{\prime}\right) ; 0\right]\left(x, t-t^{\prime}\right) d t^{\prime}\right\|_{L_{t}^{2}(0, T)} \lesssim \int_{t^{\prime}=0}^{T}\left\|\partial_{x}^{2} f\left(t^{\prime}\right)\right\|_{H^{-\frac{1}{2}\left(\mathbb{R}_{x}\right)}} d t^{\prime} \leqslant \int_{t^{\prime}=0}^{T}\left\|f\left(t^{\prime}\right)\right\|_{H^{\frac{3}{2}\left(\mathbb{R}_{x}\right)}} d t^{\prime} .
$$

Therefore, applying Cauchy-Schwarz inequality in $t^{\prime}$ we obtain

$$
\left\|\partial_{t} w(x)\right\|_{L_{t}^{2}(0, T)}^{2} \lesssim \int_{t^{\prime}=0}^{T}\left\|f\left(t^{\prime}\right)\right\|_{H^{s}\left(\mathbb{R}_{x}\right)}^{2} d t^{\prime}
$$


which combined with estimate (3.14) yields the bound (3.11b) for $s=\frac{3}{2}$.

The proof of Theorem 3.3 is complete.

The bounds of Theorem 3.3 will now be employed in the estimation of the two-dimensional forced linear Schrödinger IVP with zero initial datum:

$$
\begin{array}{ll}
i W_{t}+W_{x_{1} x_{1}}+W_{x_{2} x_{2}}=F\left(x_{1}, x_{2}, t\right), & \left(x_{1}, x_{2}, t\right) \in \mathbb{R} \times \mathbb{R} \times \mathbb{R}, \\
W\left(x_{1}, x_{2}, 0\right)=0, & \left(x_{1}, x_{2}\right) \in \mathbb{R} \times \mathbb{R},
\end{array}
$$

with solution

$$
\begin{aligned}
W\left(x_{1}, x_{2}, t\right) & =S[0 ; F]\left(x_{1}, x_{2}, t\right) \\
& =-\frac{i}{(2 \pi)^{2}} \int_{t^{\prime}=0}^{t} \int_{k_{1} \in \mathbb{R}} \int_{k_{2} \in \mathbb{R}} e^{i k_{1} x_{1}+i k_{2} x_{2}-i\left(k_{1}^{2}+k_{2}^{2}\right)\left(t-t^{\prime}\right) \widehat{F}^{x}\left(k_{1}, k_{2}, t^{\prime}\right) d k_{2} d k_{1} d t^{\prime}} \\
& =-i \int_{t^{\prime}=0}^{t} S\left[F\left(\cdot, \cdot, t^{\prime}\right) ; 0\right]\left(x_{1}, x_{2}, t-t^{\prime}\right) d t^{\prime}
\end{aligned}
$$

where $\widehat{F}$ is the Fourier transform of $F$ on the plane defined similarly to $(3.3)$ and $S\left[F\left(x_{1}, x_{2}, t^{\prime}\right) ; 0\right]$ denotes the solution of the homogeneous IVP (3.1) with initial datum $F\left(x_{1}, x_{2}, t^{\prime}\right)$.

Theorem 3.3 (Forced IVP estimates). The solution $W=S[0 ; F]$ of the forced linear Schrödinger IVP (3.19) given by (3.20) satisfies the estimates

$$
\begin{array}{ll}
\sup _{t \in[0, T]}\|W(t)\|_{H^{s}\left(\mathbb{R}_{x_{1}} \times \mathbb{R}_{x_{2}}\right)} \leqslant T \sup _{t \in[0, T]}\|F(t)\|_{H^{s}\left(\mathbb{R}_{x_{1}} \times \mathbb{R}_{x_{2}}\right)}, & s \in \mathbb{R}, \\
\sup _{x_{2} \in \mathbb{R}}\left\|W\left(x_{2}\right)\right\|_{B_{T}^{s}} \leqslant c_{s} \sqrt{T} \sup _{t \in[0, T]}\|F(t)\|_{H^{s}\left(\mathbb{R}_{x_{1}} \times \mathbb{R}_{x_{2}}\right)}, & \frac{1}{2}<s \leqslant \frac{3}{2} .
\end{array}
$$

Proof of Theorem 3.3. Estimate (3.21) can be derived by employing the Duhamel representation (3.20b) in combination with the space estimate (3.4) for the homogeneous IVP.

Concerning estimate (3.22), we recall that

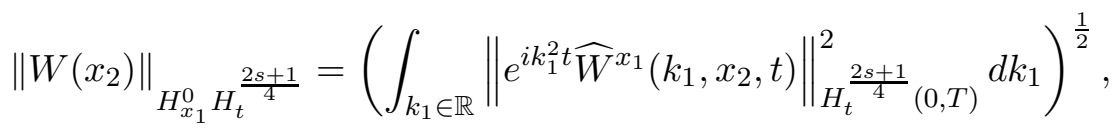

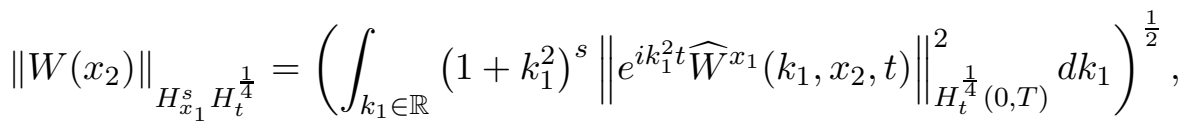

and note that the function

$$
Q\left(k_{1}, x_{2}, t\right)=e^{i k_{1}^{2} t \widehat{W}^{x_{1}}}\left(k_{1}, x_{2}, t\right)
$$

satisfies for all $k_{1} \in \mathbb{R}$ the one-dimensional IVP

$$
\begin{array}{ll}
i Q_{t}+Q_{x_{2} x_{2}}=R\left(k_{1}, x_{2}, t\right), & \left(x_{2}, t\right) \in \mathbb{R} \times \mathbb{R}, \\
Q\left(k_{1}, x_{2}, 0\right)=0, & x_{2} \in \mathbb{R},
\end{array}
$$

with forcing $R$ given by

$$
R\left(k_{1}, x_{2}, t\right)=e^{i k_{1}^{2} t} \widehat{F}^{x_{1}}\left(k_{1}, x_{2}, t\right) .
$$


Hence, for all $k_{1} \in \mathbb{R}$ the function $Q$ admits the bounds of Theorem 3.3, i.e.

$$
\begin{array}{rlrl}
\left\|Q\left(k_{1}, x_{2}\right)\right\|_{H_{t}^{\frac{2 s+1}{4}}(0, T)}^{2} \lesssim & \int_{t=0}^{T} \int_{z=0}^{T-t} \frac{1}{z^{\frac{3}{2}+s}}\left[\int_{t^{\prime}=t}^{t+z}\left\|R\left(k_{1}, t^{\prime}\right)\right\|_{H^{s}\left(\mathbb{R}_{x_{2}}\right)} d t^{\prime}\right]^{2} d z d t & \\
& +\int_{t=0}^{T}\left\|R\left(k_{1}, t\right)\right\|_{H^{s}\left(\mathbb{R}_{x_{2}}\right)}^{2} d t, & \frac{1}{2}<s<\frac{3}{2}, \\
\left\|Q\left(k_{1}, x_{2}\right)\right\|_{H_{t}^{\frac{2 s+1}{4}}(0, T)}^{2} \lesssim \int_{t=0}^{T}\left\|R\left(k_{1}, t\right)\right\|_{H^{s}\left(\mathbb{R}_{x_{2}}\right)}^{2} d t, & s=0, \frac{3}{2} .
\end{array}
$$

Combining the bound (3.24a) with the norm (3.23a) and then applying Minkowski's integral inequality in $k_{1}$ and $t^{\prime}$, we obtain

$$
\begin{aligned}
& \left\|W\left(x_{2}\right)\right\|_{H_{x_{1}}^{0} H_{t}^{\frac{2 s+1}{4}}}^{2} \lesssim \int_{t=0}^{T} \int_{z=0}^{T-t} \frac{1}{z^{\frac{3}{2}+s}}\left[\int_{t^{\prime}=t}^{t+z}\left(\int_{k_{1} \in \mathbb{R}}\left\|R\left(k_{1}, t^{\prime}\right)\right\|_{H^{s}\left(\mathbb{R}_{x_{2}}\right)}^{2} d k_{1}\right)^{\frac{1}{2}} d t^{\prime}\right]^{2} d z d t \\
& +\int_{t^{\prime}=0}^{T}\left(\int_{k_{1} \in \mathbb{R}}\left\|R\left(k_{1}, t^{\prime}\right)\right\|_{H^{s}\left(\mathbb{R}_{x_{2}}\right)}^{2} d k_{1}\right) d t^{\prime}, \quad \frac{1}{2}<s<\frac{3}{2} .
\end{aligned}
$$

Hence, noting that

$$
\int_{k_{1} \in \mathbb{R}}\left\|R\left(k_{1}, t^{\prime}\right)\right\|_{H^{s}\left(\mathbb{R}_{x_{2}}\right)}^{2} d k_{1} \leqslant\left\|F\left(t^{\prime}\right)\right\|_{H^{s}\left(\mathbb{R}_{x_{1}} \times \mathbb{R}_{x_{2}}\right)}^{2}, \quad s \geqslant 0
$$

we infer the estimate

$$
\begin{aligned}
\left\|W\left(x_{2}\right)\right\|_{H_{x_{1}}^{0} H_{t}^{\frac{2 s+1}{4}}} & \lesssim \sup _{t \in[0, T]}\|F(t)\|_{H^{s}\left(\mathbb{R}_{x_{1}} \times \mathbb{R}_{x_{2}}\right)}\left[\int_{t=0}^{T} \int_{z=0}^{T-t} \frac{1}{z^{\frac{3}{2}+s}}\left(\int_{t^{\prime}=t}^{t+z} d t^{\prime}\right)^{2} d z d t+\int_{t^{\prime}=0}^{T} d t^{\prime}\right]^{\frac{1}{2}} \\
& \lesssim \sqrt{T} \sup _{t \in[0, T]}\|F(t)\|_{H^{s}\left(\mathbb{R}_{x_{1}} \times \mathbb{R}_{x_{2}}\right)}, \quad \frac{1}{2}<s<\frac{3}{2}, x_{2} \in \mathbb{R} .
\end{aligned}
$$

In fact, starting from the bound (3.24b) and proceeding as above, we obtain the same estimate also in the case $s=\frac{3}{2}$. Therefore,

$$
\left\|W\left(x_{2}\right)\right\|_{H_{x_{1}}^{0} H_{t}^{\frac{2 s+1}{4}}} \lesssim \sqrt{T} \sup _{t \in[0, T]}\|F(t)\|_{H^{s}\left(\mathbb{R}_{x_{1}} \times \mathbb{R}_{x_{2}}\right)}, \quad \frac{1}{2}<s \leqslant \frac{3}{2}, x_{2} \in \mathbb{R} .
$$

Moreover, combining the bound (3.24b) for $s=0$ with the norm (3.23b), we find

$$
\left\|W\left(x_{2}\right)\right\|_{H_{x_{1}}^{s} H_{t}^{\frac{1}{4}}}^{2} \lesssim \int_{t=0}^{T} \int_{k_{1} \in \mathbb{R}}\left(1+k_{1}^{2}\right)^{s}\left\|e^{i k_{1}^{2} t} \widehat{F}^{x_{1}}\left(k_{1}, x_{2}, t\right)\right\|_{L^{2}\left(\mathbb{R}_{x_{2}}\right)}^{2} d k_{1} d t
$$

so by Parseval-Plancherel in $x_{2}$ and $k_{2}$ we infer

$$
\begin{aligned}
\left\|W\left(x_{2}\right)\right\|_{H_{x_{1}}^{s} H_{t}^{\frac{1}{4}}} & \lesssim\left(\int_{t^{\prime}=0}^{T}\left\|F\left(t^{\prime}\right)\right\|_{H^{s}\left(\mathbb{R}_{x_{1}} \times \mathbb{R}_{x_{2}}\right)}^{2} d t^{\prime}\right)^{\frac{1}{2}} \\
& \leqslant \sqrt{T} \sup _{t \in[0, T]}\|F(t)\|_{H^{s}\left(\mathbb{R}_{x_{1}} \times \mathbb{R}_{x_{2}}\right)}, \quad s \geqslant 0 .
\end{aligned}
$$

Estimates (3.25) and (3.26) imply estimate (3.22) via the definition of the $B_{T}^{s}$-norm. 


\section{The Forced Linear IBVP: Proof of Theorem 1.2}

We shall now combine the pure IBVP Theorem 2.1 with the IVP Theorems 3.1 and 3.3 in order to estimate the solution of the forced linear IBVP (1.6). To do so, we first decompose this problem into simpler problems that have already been estimated in the preceding sections.

Decomposition into simpler problems. IBVP (1.6) can be expressed as the superposition of the homogeneous linear IBVP

$$
\begin{array}{ll}
i u_{t}+u_{x_{1} x_{1}}+u_{x_{2} x_{2}}=0, & \left(x_{1}, x_{2}, t\right) \in \mathbb{R} \times \mathbb{R}^{+} \times(0, T), \\
u\left(x_{1}, x_{2}, 0\right)=u_{0}\left(x_{1}, x_{2}\right) \in H^{s}\left(\mathbb{R}_{x_{1}} \times \mathbb{R}_{x_{2}}^{+}\right), & \left(x_{1}, x_{2}\right) \in \mathbb{R} \times \mathbb{R}^{+}, \\
u\left(x_{1}, 0, t\right)=g_{0}\left(x_{1}, t\right) \in B_{T}^{s}, & \left(x_{1}, t\right) \in \mathbb{R} \times[0, T],
\end{array}
$$

and the forced linear IBVP with zero initial and boundary data

$$
\begin{array}{ll}
i u_{t}+u_{x_{1} x_{1}}+u_{x_{2} x_{2}}=f \in C\left([0, T] ; H^{s}\left(\mathbb{R}_{x_{1}} \times \mathbb{R}_{x_{2}}^{+}\right)\right), & \left(x_{1}, x_{2}, t\right) \in \mathbb{R} \times \mathbb{R}^{+} \times(0, T), \\
u\left(x_{1}, x_{2}, 0\right)=0, & \left(x_{1}, x_{2}\right) \in \mathbb{R} \times \mathbb{R}^{+}, \\
u\left(x_{1}, 0, t\right)=0, & \left(x_{1}, t\right) \in \mathbb{R} \times[0, T] .
\end{array}
$$

The above decomposition has decoupled the forcing $f$ from the data $u_{0}, g_{0}$. Next, we will perform further decompositions in order to separate the data from each other.

In particular, let the initial datum $U_{0}$ of the homogeneous linear IVP (3.1) be defined as a whole-plane extension of the half-plane initial datum $u_{0}$ of IBVP (4.1) such that

$$
\left\|U_{0}\right\|_{H^{s}\left(\mathbb{R}_{x_{1}} \times \mathbb{R}_{x_{2}}\right)} \leqslant c\left\|u_{0}\right\|_{H^{s}\left(\mathbb{R}_{x_{1}} \times \mathbb{R}_{x_{2}}^{+}\right)} .
$$

Then, problem (4.1) can be expressed as the superposition of IVP (3.1) and the following homogeneous linear IBVP with zero initial datum:

$$
\begin{array}{ll}
i u_{t}+u_{x_{1} x_{1}}+u_{x_{2} x_{2}}=0, & \left(x_{1}, x_{2}, t\right) \in \mathbb{R} \times \mathbb{R}^{+} \times(0, T), \\
u\left(x_{1}, x_{2}, 0\right)=0, & \left(x_{1}, x_{2}\right) \in \mathbb{R} \times \mathbb{R}^{+}, \\
u\left(x_{1}, 0, t\right)=G_{0}\left(x_{1}, t\right)=g_{0}\left(x_{1}, t\right)-U\left(x_{1}, 0, t\right), & \left(x_{1}, t\right) \in \mathbb{R} \times[0, T],
\end{array}
$$

where the function $U\left(x_{1}, 0, t\right)$ involved in the boundary condition is the solution $U=S\left[U_{0} ; 0\right]$ of $\operatorname{IVP}(3.1)$ evaluated at $x_{2}=0$ (this trace is well-defined since $s>\frac{1}{2}$ ).

In addition, let the forcing $F$ of the forced linear IVP (3.19) be defined as a whole-plane extension of the half-plane forcing $f$ of IBVP (1.6) such that

$$
\|F\|_{C\left([0, T] ; H^{s}\left(\mathbb{R}_{x_{1}} \times \mathbb{R}_{x_{2}}\right)\right)} \leqslant c\|f\|_{C\left([0, T] ; H^{s}\left(\mathbb{R}_{x_{1}} \times \mathbb{R}_{x_{2}}^{+}\right)\right)} .
$$

Then, problem (4.2) can be written as the superposition of IVP (3.19) and the following homogeneous linear IBVP with zero initial datum:

$$
\begin{array}{ll}
i u_{t}+u_{x_{1} x_{1}}+u_{x_{2} x_{2}}=0, & \left(x_{1}, x_{2}, t\right) \in \mathbb{R} \times \mathbb{R}^{+} \times(0, T), \\
u\left(x_{1}, x_{2}, 0\right)=0, & \left(x_{1}, x_{2}\right) \in \mathbb{R} \times \mathbb{R}^{+}, \\
u\left(x_{1}, 0, t\right)=W\left(x_{1}, 0, t\right), & \left(x_{1}, t\right) \in \mathbb{R} \times[0, T],
\end{array}
$$

where the boundary datum $W\left(x_{1}, 0, t\right)$ is obtained by evaluating the solution $W=S[0 ; F]$ of IVP (3.19) at $x_{2}=0$ (this trace is well-defined since $s>\frac{1}{2}$ ). 
In summary, the solution of the forced linear IBVP (1.6) can be analyzed into the respective solutions of the four component problems (3.1), (3.19), (4.4) and (4.6) involved in the above decompositions as follows:

$$
S\left[u_{0}, g_{0} ; f\right]=\left.S\left[U_{0} ; 0\right]\right|_{x_{2} \in \mathbb{R}^{+}}+\left.S[0 ; F]\right|_{x_{2} \in \mathbb{R}^{+}}+S\left[0, G_{0} ; 0\right]-S\left[0,\left.W\right|_{x_{2}=0} ; 0\right] .
$$

The first two terms on the right-hand side of (4.7) have been estimated in Theorems 3.1 and 3.3 respectively. Also, the remaining two terms can be estimated via Theorem 2.1 as their associated problems, namely IBVPs (4.4) and (4.6), essentially correspond to different versions of the pure IBVP (2.1). Indeed, both of these problems are homogeneous, with zero initial datum, and with boundary datum in $B_{T}^{s}$ since for $\frac{1}{2}<s \leqslant \frac{3}{2}$ estimates (3.5), (3.22), (4.3) and (4.5) imply

$$
\begin{aligned}
& \left\|G_{0}\right\|_{B_{T}^{s}} \lesssim\left\|g_{0}\right\|_{B_{T}^{s}}+\left\|u_{0}\right\|_{H^{s}\left(\mathbb{R}_{x_{1}} \times \mathbb{R}_{x_{2}}^{+}\right)}, \\
& \left\|\left.W\right|_{x_{2}=0}\right\|_{B_{T}^{s}} \lesssim \sqrt{T} \sup _{t \in[0, T]}\|f(t)\|_{H^{s}\left(\mathbb{R}_{x_{1}} \times \mathbb{R}_{x_{2}}^{+}\right)} .
\end{aligned}
$$

Moreover, thanks to the compatibility condition (1.5) and the initial conditions (3.1b) and (3.19b), for $s>1$ the boundary data $G_{0}$ and $\left.W\right|_{x_{2}=0}$ both vanish at $t=0$ for all $x_{1} \in \mathbb{R}$.

Hence, restricting $1<s \leqslant \frac{3}{2}$, we can treat IBVPs (4.4) and (4.6) simultaneously by considering the following problem:

$$
\begin{array}{ll}
i u_{t}+u_{x_{1} x_{1}}+u_{x_{2} x_{2}}=0, & \left(x_{1}, x_{2}, t\right) \in \mathbb{R} \times \mathbb{R}^{+} \times(0, T), \\
u\left(x_{1}, x_{2}, 0\right)=0, & \left(x_{1}, x_{2}\right) \in \mathbb{R} \times \overline{\mathbb{R}^{+}}, \\
u\left(x_{1}, 0, t\right)=Q_{0}\left(x_{1}, t\right) \in B_{T}^{s}, & \left(x_{1}, t\right) \in \mathbb{R}_{x_{1}} \times[0, T],
\end{array}
$$

where the boundary datum satisfies the compatibility condition

$$
Q_{0}\left(x_{1}, 0\right)=0 \quad \forall x_{1} \in \mathbb{R} .
$$

Next, we will reduce the estimation of IBVP (4.10) to that of the pure IBVP (2.1). Let

$$
q_{0}\left(k_{1}, t\right)=e^{i k_{1}^{2} t}{\widehat{Q_{0}}}^{x_{1}}\left(k_{1}, t\right)
$$

and note that $q_{0} \in H_{t}^{\frac{2 s+1}{4}}(0, T)$ since $Q_{0} \in B_{T}^{s}$. Moreover, $q_{0}\left(k_{1}, 0\right)=0$ for all $k_{1} \in \mathbb{R}$ thanks to condition (4.11). Let $E$ be an extension of $q_{0}$ from $[0, T]$ to $\mathbb{R}_{t}$ such that

$$
\left\|E\left(k_{1}\right)\right\|_{H^{\frac{2 s+1}{4}}\left(\mathbb{R}_{t}\right)} \leqslant c\left\|q_{0}\left(k_{1}\right)\right\|_{H_{t}^{\frac{2 s+1}{4}}(0, T)}, \quad c>0 .
$$

Consider the function $E_{\theta}\left(k_{1}, t\right)=\theta(t) E\left(k_{1}, t\right) \in H^{\frac{2 s+1}{4}}\left(\mathbb{R}_{t}\right)$, where $\theta \in C_{0}^{\infty}(\mathbb{R})$ with $\theta=1$ on $[-1,1]$, $\theta=0$ on $(-2,2)^{c}$ and $\|\theta\|_{L^{\infty}(\mathbb{R})}=1$. Since $T<1$, we have $E_{\theta}=q$ on $\mathbb{R}_{k_{1}} \times[0, T]$. Furthermore, combining the algebra property in $H^{\frac{2 s+1}{4}}\left(\mathbb{R}_{t}\right)$ with estimate $(4.13)$, we find

$$
\left\|E_{\theta}\left(k_{1}\right)\right\|_{H^{\frac{2 s+1}{4}}\left(\mathbb{R}_{t}\right)} \leqslant c_{s}\left\|q_{0}\left(k_{1}\right)\right\|_{H_{t}^{\frac{2 s+1}{4}}(0, T)}, \quad k_{1} \in \mathbb{R} .
$$

Also, $E\left(k_{1}, 0\right)=q_{0}\left(k_{1}, 0\right)=0$ and $\operatorname{supp}\left(E_{\theta}\right) \subset \mathbb{R}_{k_{1}} \times(-2,2)$ so that, in particular, $E_{\theta}\left(k_{1}, 2\right)=0$. Thus, $E_{\theta} \in H_{0}^{\frac{2 s+1}{4}}(0,2)$ and hence Theorem 11.4 of [LM] implies that the extension

$$
q\left(k_{1}, t\right)= \begin{cases}E_{\theta}\left(k_{1}, t\right), & t \in(0,2) \\ 0, & t \in(0,2)^{c}\end{cases}
$$


belongs to $H^{\frac{2 s+1}{4}}\left(\mathbb{R}_{t}\right)$ with $\left\|q\left(k_{1}\right)\right\|_{H^{\frac{2 s+1}{4}}\left(\mathbb{R}_{t}\right)} \leqslant c_{s}\left\|E_{\theta}\left(k_{1}\right)\right\|_{H_{0}^{\frac{2 s+1}{4}}(0,2)}$ for all $k_{1} \in \mathbb{R}$ and $1<s \leqslant \frac{3}{2}$. Therefore, recalling estimate (4.14) we infer

$$
\left\|q\left(k_{1}\right)\right\|_{H^{\frac{2 s+1}{4}}\left(\mathbb{R}_{t}\right)} \leqslant c_{s}\left\|q_{0}\left(k_{1}\right)\right\|_{H_{t}^{\frac{2 s+1}{4}}(0, T)}, \quad 1<s \leqslant \frac{3}{2}, k_{1} \in \mathbb{R},
$$

Overall, we have constructed an extension $q$ of $q_{0}$ that satisfies the bound (4.16) and has support $\operatorname{supp}(q) \subset \mathbb{R}_{k_{1}} \times(0,2)$. Also, again by Theorem 11.4 of $[\mathrm{LM}]$, the extension $\widetilde{q}$ of $q$ by zero outside $[0, T]$ satisfies

$$
\left\|\widetilde{q}\left(k_{1}\right)\right\|_{H^{\frac{1}{4}\left(\mathbb{R}_{t}\right)}} \leqslant c\left\|q_{0}\left(k_{1}\right)\right\|_{H_{t}^{\frac{1}{4}}(0, T)}, \quad k_{1} \in \mathbb{R} .
$$

Thus, letting the boundary datum $g$ of the pure IBVP (2.1) be given by

$$
\widehat{g}^{x_{1}}\left(k_{1}, t\right)=e^{i k_{1}^{2} t} q\left(k_{1}, t\right)=e^{i k_{1}^{2} t} \widetilde{q}\left(k_{1}, t\right), \quad\left(k_{1}, t\right) \in \mathbb{R} \times[0,2],
$$

and recalling (4.12), we conclude that IBVP (4.10) is embedded inside the pure IBVP (2.1), that is $S\left[0, Q_{0} ; 0\right]=\left.S[0, g ; 0]\right|_{t \in[0, T]}$. Also, importantly, the above construction together with (4.12) and (4.18) guarantees that

$$
\|g\|_{B^{s}} \lesssim\left\|Q_{0}\right\|_{B_{T}^{s}}, \quad 1<s \leqslant \frac{3}{2}
$$

Therefore, thanks to Theorem 2.1 we deduce the estimate

$$
\sup _{t \in[0, T]}\left\|S\left[0, Q_{0} ; 0\right](t)\right\|_{H^{s}\left(\mathbb{R}_{x_{1}} \times \mathbb{R}_{x_{2}}^{+}\right)} \lesssim\left\|Q_{0}\right\|_{B_{T}^{s}}, \quad 1<s \leqslant \frac{3}{2} .
$$

Proof of Theorem 1.2. At this point, all four components of the superposition (4.7) have been estimated. In particular, estimate (3.4) of Theorem 3.1 and inequality (4.3) imply

$$
\sup _{t \in[0, T]}\left\|S\left[U_{0} ; 0\right](t)\right\|_{H^{s}\left(\mathbb{R}_{x_{1}} \times \mathbb{R}_{x_{2}}\right)} \leqslant c\left\|u_{0}\right\|_{H^{s}\left(\mathbb{R}_{x_{1}} \times \mathbb{R}_{x_{2}}^{+}\right)}, \quad s \in \mathbb{R}
$$

estimate (3.21) of Theorem 3.3 together with inequality (4.5) and the fact that $T<1$ yield

$$
\sup _{t \in[0, T]}\|S[0 ; F](t)\|_{H^{s}\left(\mathbb{R}_{x_{1}} \times \mathbb{R}_{x_{2}}\right)} \leqslant c \sqrt{T} \sup _{t \in[0, T]}\|f(t)\|_{H^{s}\left(\mathbb{R}_{x_{1}} \times \mathbb{R}_{x_{2}}^{+}\right)}, \quad s \in \mathbb{R},
$$

estimate (4.20) and inequality (4.8) with $Q_{0}=G_{0}$ imply

$$
\sup _{t \in[0, T]}\left\|S\left[0, G_{0} ; 0\right](t)\right\|_{H^{s}\left(\mathbb{R}_{x_{1}} \times \mathbb{R}_{x_{2}}^{+}\right)} \leqslant c_{s}\left(\left\|u_{0}\right\|_{H^{s}\left(\mathbb{R}_{x_{1}} \times \mathbb{R}_{x_{2}}^{+}\right)}+\left\|g_{0}\right\|_{B_{T}^{s}}\right), \quad 1<s \leqslant \frac{3}{2},
$$

and estimate (4.20) together with inequality (4.9) with $Q_{0}=\left.W\right|_{x_{2}=0}$ yield

$$
\sup _{t \in[0, T]}\left\|S\left[0,\left.W\right|_{x_{2}=0} ; 0\right](t)\right\|_{H^{s}\left(\mathbb{R}_{x_{1}} \times \mathbb{R}_{x_{2}}^{+}\right)} \leqslant c_{s} \sqrt{T} \sup _{t \in[0, T]}\|f(t)\|_{H^{s}\left(\mathbb{R}_{x_{1}} \times \mathbb{R}_{x_{2}}^{+}\right)}, \quad 1<s \leqslant \frac{3}{2} .
$$

Combining the above four estimates, we deduce estimate (1.10) for the forced linear Schrödinger $\operatorname{IBVP}(1.6)$.

\section{Well-Posedness of NLS on the Half-Plane: Proof of Theorem 1.1}

Using the forced linear IBVP estimate (1.10) and the contraction mapping theorem, we shall now establish well-posedness of the NLS IBVP (1.1) in the sense of Hadamard, i.e. we shall show that there exists a unique solution to this problem that depends continuously on the initial and boundary data. 
Existence and uniqueness. Setting $f= \pm|u|^{p-1} u$ in the unified transform formula (1.7) for the solution $S\left[u_{0}, g_{0} ; f\right]$ of the forced linear IBVP (1.6) gives rise to the following iteration map for the NLS IBVP (1.1):

$$
u \mapsto \Phi u=\Phi_{u_{0}, g_{0}} u=S\left[u_{0}, g_{0} ; \pm|u|^{p-1} u\right] .
$$

We shall show that this map is a contraction in the space

$$
X=C\left(\left[0, T^{*}\right] ; H^{s}\left(\mathbb{R}_{x_{1}} \times \mathbb{R}_{x_{2}}^{+}\right)\right)
$$

for some appropriate lifespan $T^{*} \in(0, T]$ to be determined.

Showing that the map $u \mapsto \Phi u$ is onto $X$. For $1<s \leqslant \frac{3}{2}$, estimate (1.10) implies

$$
\|\Phi u\|_{X} \leqslant c_{s}\left(\left\|u_{0}\right\|_{H^{s}\left(\mathbb{R}_{x_{1}} \times \mathbb{R}_{x_{2}}^{+}\right)}+\left\|g_{0}\right\|_{B_{T}^{s}}+\sqrt{T^{*}} \sup _{t \in\left[0, T^{*}\right]}\left\|(u \bar{u})^{\frac{p-1}{2}}(t)\right\|_{H^{s}\left(\mathbb{R}_{x_{1}} \times \mathbb{R}_{x_{2}}^{+}\right)}\right),
$$

where we have written $|u|^{p-1}=(u \bar{u})^{\frac{p-1}{2}}$ since $\frac{p-1}{2} \in \mathbb{N}$. Thus, by repeated use of the algebra property in $H^{s}\left(\mathbb{R}_{x_{1}} \times \mathbb{R}_{x_{2}}^{+}\right)$we obtain

$$
\|\Phi u\|_{X} \leqslant c_{s}\left(\left\|u_{0}\right\|_{H^{s}\left(\mathbb{R}_{x_{1}} \times \mathbb{R}_{x_{2}}^{+}\right)}+\left\|g_{0}\right\|_{B_{T}^{s}}+\sqrt{T^{*}}\|u\|_{X}^{p}\right) .
$$

Let $B(0, r)=\left\{u \in X:\|u\|_{X} \leqslant r\right\}$ be a ball centered at 0 with radius $r=2 c_{s}\left\|\left(u_{0}, g_{0}\right)\right\|_{D}=$ $\left\|u_{0}\right\|_{H^{s}\left(\mathbb{R}_{x_{1}} \times \mathbb{R}_{x_{2}}^{+}\right)}+\left\|g_{0}\right\|_{B_{T}^{s}}$. For $u \in B(0, r)$, estimate (5.3) implies

$$
\|\Phi u\|_{X} \leqslant \frac{r}{2}+c_{s} \sqrt{T^{*}} r^{p} .
$$

Hence, choosing

$$
T^{*} \leqslant \frac{1}{4 c_{s}^{2} r^{2(p-1)}}
$$

ensures that $\Phi u \in B(0, r)$ whenever $u \in B(0, r)$.

Showing that the map $u \mapsto \Phi u$ is a contraction in $X$. We shall show that

$$
\left\|\Phi u_{1}-\Phi u_{2}\right\|_{X} \leqslant \frac{1}{2}\left\|u_{1}-u_{2}\right\|_{X}
$$

for any $u_{1}, u_{2} \in B(0, r)$ and $1<s \leqslant \frac{3}{2}$. Noting that

$$
\Phi u_{1}-\Phi u_{2}=S\left[0,0 ; \pm\left(\left|u_{1}\right|^{p-1} u_{1}-\left|u_{2}\right|^{p-1} u_{2}\right)\right]
$$

and using estimate (1.10), we obtain

$$
\left\|\Phi u_{1}-\Phi u_{2}\right\|_{X} \leqslant c_{s} \sqrt{T^{*}} \sup _{t \in\left[0, T^{*}\right]}\left\|\left(\left|u_{1}\right|^{p-1} u_{1}-\left|u_{2}\right|^{p-1} u_{2}\right)(t)\right\|_{H^{s}\left(\mathbb{R}_{x_{1}} \times \mathbb{R}_{x_{2}}^{+}\right)} .
$$

Since $\frac{p-1}{2} \in \mathbb{N}$, we can write $\left|u_{1}\right|^{p-1} u_{1}-\left|u_{2}\right|^{p-1} u_{2}=\left(u_{1} \bar{u}_{1}\right)^{\frac{p-1}{2}} u_{1}-\left(u_{2} \bar{u}_{2}\right)^{\frac{p-1}{2}} u_{2}$, which is a polynomial that vanishes when $u_{1}=u_{2}$. Hence, we must have

$$
\left|u_{1}\right|^{p-1} u_{1}-\left|u_{2}\right|^{p-1} u_{2}=P\left(u_{1}, \bar{u}_{1}, u_{2}, \bar{u}_{2}\right)\left(u_{1}-u_{2}\right)+Q\left(u_{1}, \bar{u}_{1}, u_{2}, \bar{u}_{2}\right)\left(\overline{u_{1}-u_{2}}\right),
$$

for some polynomials $P$ and $Q$ of degree $(p-1)$.

Combining inequality (5.6) with the writing (5.7) and the algebra property in $H^{s}\left(\mathbb{R}_{x_{1}} \times \mathbb{R}_{x_{2}}^{+}\right)$, we find

$$
\begin{aligned}
\left\|\Phi u_{1}-\Phi u_{2}\right\|_{X} & \leqslant c_{s} \sqrt{T^{*}}\left(\left\|P\left(u_{1}, \bar{u}_{1}, u_{2}, \bar{u}_{2}\right)\right\|_{X}+\left\|Q\left(u_{1}, \bar{u}_{1}, u_{2}, \bar{u}_{2}\right)\right\|_{X}\right)\left\|u_{1}-u_{2}\right\|_{X} \\
& \leqslant c_{s} \sqrt{T^{*}} c_{p} r^{p-1}\left\|u_{1}-u_{2}\right\|_{X}, \quad c_{p}>1 .
\end{aligned}
$$


Hence, choosing

$$
T^{*} \leqslant \frac{1}{4 c_{p}^{2} c_{s}^{2} r^{2(p-1)}}
$$

ensures that the contraction inequality (5.5) is satisfied for all $u_{1}, u_{2} \in B(0, r)$.

Note that, since $c_{p}>1$, the contraction condition (5.8) is stronger than the onto condition (5.4). Overall, for $T^{*} \in(0, T]$ satisfying (5.8), the map $u \mapsto \Phi u$ defined by (5.1) is both onto and a contraction on the ball $B(0, r)$. Hence, by the contraction mapping theorem it follows that $u \mapsto \Phi u$ has a unique fixed point in $B(0, r)$. Equivalently, the integral equation $u=\Phi u$ for the solution of the NLS IBVP (1.1) has a unique solution $u \in B(0, r) \subset X$.

Continuity of the data-to-solution map. Finally, we shall show that the data-to-solution map

$$
H^{s}\left(\mathbb{R}_{x_{1}} \times \mathbb{R}_{x_{2}}^{+}\right) \times B_{T}^{s} \ni\left(u_{0}, g_{0}\right) \mapsto u \in X
$$

is continuous.

Let $\left(u_{0}, g_{0}\right)$ and $\left(w_{0}, h_{0}\right)$ be two pairs of data lying inside a ball $B_{\varrho} \subset D$ of radius $\varrho>0$ centered at a distance $r$ from 0 . Denote by $u=\Phi_{u_{0}, g_{0}} u$ and $w=\Phi_{w_{0}, h_{0}} w$ the corresponding solutions to the NLS IBVP (1.1) and by $T_{u}$ and $T_{w}$ their respective lifespans, which are given by

$T_{u}=\min \left\{T, c_{s, p}\left\|\left(u_{0}, g_{0}\right)\right\|_{D}^{-2(p-1)}\right\}, \quad T_{w}=\min \left\{T, c_{s, p}\left\|\left(w_{0}, h_{0}\right)\right\|_{D}^{-2(p-1)}\right\}, \quad c_{s, p}=\left(2^{2 p} c_{p}^{2} c_{s}^{2 p}\right)^{-1}$.

Since $\max \left\{\left\|\left(u_{0}, g_{0}\right)\right\|_{D},\left\|\left(w_{0}, h_{0}\right)\right\|_{D}\right\} \leqslant r+\varrho$ and $p>1$, it follows that

$$
\min \left\{T_{u}, T_{w}\right\} \geqslant \min \left\{T, c_{s, p}(r+\varrho)^{-2(p-1)}\right\}=T_{c} .
$$

Hence, both solutions are guaranteed to exist for $0 \leqslant t \leqslant T_{c}$.

The common lifespan $T_{c}$ gives rise to the space $X_{c}=C\left(\left[0, T_{c}\right] ; H^{s}\left(\mathbb{R}_{x_{1}} \times \mathbb{R}_{x_{2}}^{+}\right)\right)$. We shall now determine the radius $r_{c}$ of a ball $B\left(0, r_{c}\right) \subset X_{c}$ such that $u, w \in B\left(0, r_{c}\right)$ and

$$
\|u-w\|_{X_{c}} \leqslant 2 c_{s}\left\|\left(u_{0}-w_{0}, g_{0}-h_{0}\right)\right\|_{D} .
$$

Recall that $u$ and $w$ are fixed points of the map $\Phi$ in the spaces $X_{u}$ and $X_{w}$ defined by (5.2) with $T^{*}$ replaced by $T_{u}$ and $T_{w}$ respectively. Hence, since $X_{u}, X_{w} \subset X_{c}$ by the definition of $T_{c}, u-w$ is a fixed point of $\Phi$ in $X_{c}$. Thus, using estimate (1.10) together with the writing (5.7) and the algebra property in $H^{s}\left(\mathbb{R}_{x_{1}} \times \mathbb{R}_{x_{2}}^{+}\right)$, we obtain

$$
\begin{aligned}
\|u-w\|_{X_{c}} & =\left\|S\left[u_{0}-w_{0}, g_{0}-h_{0}, \pm\left(|u|^{p-1} u-w|w|^{p-1}\right)\right]\right\|_{X_{c}} \\
& \leqslant c_{s}\left\|\left(u_{0}-w_{0}, g_{0}-h_{0}\right)\right\|_{D}+c_{s} c_{p} \sqrt{T_{c}} r_{c}^{p-1}\|u-w\|_{X_{c}} .
\end{aligned}
$$

For

$$
r_{c}=\left(2 c_{s} c_{p} \sqrt{T_{c}}\right)^{-\frac{1}{p-1}}=\max \left\{\left(2 c_{s} c_{p} \sqrt{T}\right)^{-\frac{1}{p-1}}, 2 c_{s}(r+\varrho)\right\}
$$

estimate (5.12) implies inequality (5.11) which in turn establishes local Lipschitz continuity of the data-to-solution map. The proof of Theorem 1.1 is complete.

Remark 5.1. Estimates (2.4), (3.5) and (3.22) give rise to the following additional estimate for the forced IBVP (1.6):

$$
\sup _{x_{2} \in \mathbb{R}^{+}}\left\|u\left(x_{2}\right)\right\|_{B_{T}^{s}} \leqslant c_{s}\left(\left\|u_{0}\right\|_{H^{s}\left(\mathbb{R}_{x_{1}} \times \mathbb{R}_{x_{2}}^{+}\right)}+\left\|g_{0}\right\|_{B_{T}^{s}}+\sqrt{T} \sup _{t \in[0, T]}\|f(t)\|_{H^{s}\left(\mathbb{R}_{x_{1}} \times \mathbb{R}_{x_{2}}^{+}\right)}\right), \quad 1<s \leqslant \frac{3}{2},
$$

which, as noted in Remark 2.1, can be combined with estimate (1.10) in order to show well-posedness of the NLS IBVP $(1.1)$ in the smaller space $C\left(\left[0, T^{*}\right] ; H^{s}\left(\mathbb{R}_{x_{1}} \times \mathbb{R}_{x_{2}}^{+}\right)\right) \cap C\left(\mathbb{R}_{x_{2}}^{+} ; B_{T^{*}}^{s}\right)$ instead of the Hadamard space of Theorem 1.1. 
Acknowledgements. This work was partially supported by a grant from the Simons Foundation (\#524469 to Alex Himonas). The authors are grateful to Athanassios Fokas for being the catalyst of this project and for making valuable suggestions that led to the improvement of this work.

\section{REFERENCES}

[AKNS] M. Ablowitz, D. Kaup, A. Newel and H. Segur, Inverse scattering transform - Fourier analysis for nonlinear problems. Stud. Appl. Math. 53 (1974), 249-315.

[AG] G. Agarwal, Nonlinear fiber optics, Academic Press, 2007.

[AU] C. Audiard, Global Strichartz estimates for the Schrödinger equation with non zero boundary conditions and applications. arXiv:1702.06792v1 (2017).

[BM] G. Biondini and D. Mantzavinos, Long-time asymptotics for the focusing nonlinear Schrödinger equation with nonzero boundary conditions at infinity and asymptotic stage of modulational instability. Comm. Pure Appl. Math. 70 (2017), 2300-2365.

[BSZ1] J. Bona, S. Sun and B.-Y. Zhang, A non-homogeneous boundary-value problem for the Korteweg-de Vries equation in a quarter plane. Trans. Amer. Math. Soc. 354 (2002), 427-490.

[BSZ2] J. Bona, S. Sun and B.-Y. Zhang, Nonhomogeneous boundary value problems of one-dimensional nonlinear Schrödinger equations. J. Math. Pures Appl. 109 (2018), 1-66.

[B1] J. Bourgain, Fourier transform restriction phenomena for certain lattice subsets and applications to nonlinear evolution equations. Geom. Funct. Anal. 3 (1993), 107-157.

[B2] J. Bourgain, Refinements of Strichartz' inequality and applications to 2D-NLS with critical non-linearity. Int. Math. Res. Not. 1998 (1998), 253-283.

[B3] J. Bourgain, Global well-posedness of defocusing critical nonlinear Schrödinger equation in the radial case. J. Amer. Math. Soc. 12 (1999), 145-171.

[B4] J. Bourgain, Global solutions of nonlinear Schrödinger equations, AMS Colloquium Publications 46, AMS, Providence, RI, 1999.

[BG] H. Brezis and T. Gallouet, Nonlinear Schrödinger evolution equations. Nonlinear Anal. 4 (1980), 677-681.

[BGT1] N. Burq, P. Gérard and N. Tzvetkov, Two singular dynamics of the nonlinear Schrödinger equation on a plane domain. Geom. Funct. Anal. 13 (2003), 1-19.

[BGT2] N. Burq, P. Gérard and N. Tzvetkov, On nonlinear Schrödinger equations in exterior domains. Ann. Inst. H. Poincaré Anal. Non Linéaire 21 (2004), 295-318.

[BGT3] N. Burq, P. Gérard and N. Tzvetkov, Strichartz inequalities and the nonlinear Schrödinger equation on compact manifolds. Amer. J. Math. 126 (2004), 569-605.

[CW1] T. Cazenave and F. Weissler, Some remarks on the nonlinear Schrödinger equation in the critical case. Nonlinear Semigroups, Partial Differential Equations, and Attractors, Lecture Notes in Mathematics 1394 (1989), 18-29.

[CW2] T. Cazenave and F. Weissler, The Cauchy problem for the critical nonlinear Schrödinger equation in $H^{s}$. Nonlinear Anal. 14 (1990), 807-836.

[CP] T. Chen and N. Pavlovic, The quintic NLS as the mean field limit of a boson gas with three-body interactions. J. Funct. Anal. 260 (2011), 959-997.

$[\mathrm{CH}] \quad \mathrm{X}$. Chen and J. Holmer, On the rigorous derivation of the $2 D$ cubic nonlinear Schrödinger equation from $3 D$ quantum many-body dynamics. Arch. Ration. Mech. Anal. 210 (2013), 909-954.

[CK] J. Colliander and C. Kenig, The generalized Korteweg-de Vries equation on the half-line. Comm. Partial Differential Equations 27 (2002), 2187-2266.

[CKSTT] J. Colliander, M. Keel, G. Staffilani, H. Takaoka and T. Tao, Global well-posedness and scattering in the energy space for the critical nonlinear Schrödinger equation in $\mathbb{R}^{3}$. Ann. of Math. 167 (2008), 767-865.

[CS] P. Constantin and J.-C. Saut, Local smoothing properties of Schrödinger equations. Indiana U. Math. J. 38 (1989), 781-810.

[CSS] W. Craig, C. Sulem and P.-L. Sulem, Nonlinear modulation of gravity waves: a rigorous approach. Nonlinearity 5 (1992), 497-522.

[CKS] W. Craig, T. Kappeler and W. Strauss, Microlocal dispersive smoothing for the Schrödinger equation. Comm. Pure Appl. Math. 48 (1995), 769-860.

[DZ] P. Deift and X. Zhou, Long-time behavior of the non-focusing nonlinear Schrödinger equation - a case study, New Series, Lectures in Mathematical Sciences 5, University of Tokyo, Tokyo, 1995. 
[D] B. Dodson, Global well-posedness and scattering for the defocusing, $L^{2}$-critical nonlinear Schrödinger equation when $d \geqslant 3$. J. Amer. Math. Soc. 25 (2012), 429-463.

[F1] A. Fokas, A unified transform method for solving linear and certain nonlinear PDEs. Proc. R. Soc. A 453 (1997), 1411-1443.

[F2] A. Fokas, A unified approach to boundary value problems, SIAM, 2008.

[F3] A. Fokas, A new transform method for evolution partial differential equations. IMA Journal of Applied Mathematics 67 (2002), 559-590.

[F4] A. Fokas, The Davey-Stewartson equation on the half-plane. Comm. Math. Phys. 289 (2009), 957-993.

$[\mathrm{FH}] \quad$ A. Fokas and A. Himonas, Well-posedness of an integrable generalization of the nonlinear Schrödinger equation on the circle. Lett. Math. Phys. 96 (2011), 169-189.

[FHM1] A. Fokas, A. Himonas and D. Mantzavinos, The nonlinear Schrödinger equation on the half-line. Trans. Amer. Math. Soc. 369 (2017), 681-709.

[FHM2] A. Fokas, A. Himonas and D. Mantzavinos, The Korteweg-de Vries equation on the half-line. Nonlinearity 29 (2016), 489-527.

[FI] A. Fokas and A. Its, The nonlinear Schrödinger equation on the interval. J. Phys. A: Math. Gen. 37 (2004), 6091-6114.

[FIS] A. Fokas, A. Its and L.-Y. Sung, The nonlinear Schrödinger equation on the half-line. Nonlinearity 18 (2005), 1771-1822.

[FP] A. Fokas and B. Pelloni, A transform method for linear evolution PDEs on a finite interval. IMA J. Appl. Math. 70 (2005), 564-587.

[FS] A. Fokas and E. Spence, Synthesis, as opposed to separation, of variables. SIAM Rev. 54 (2012), 291-324.

[GS] J. Ghidaglia and J.-C. Saut, Nonelliptic Schrödinger equations. J. Nonlin. Sci. 3 (1993), $169-195$.

[GV1] J. Ginibre and G. Velo, On a class of nonlinear Schrödinger equations. II. Scattering theory, general case. J. Funct. Anal. 32 (1979), 33-71.

[GV2] J. Ginibre and G. Velo, Scattering theory in the energy space for a class of nonlinear Schrödinger equations. J. Math. Pure Appl. 64 (1985), 363-401.

[HO] H. Hasimoto and H. Ono, Nonlinear modulation of gravity waves. J. Phys. Soc. Japan 33 (1972), 805-811.

[HM] A. Himonas and D. Mantzavinos, The "good" Boussinesq equation on the half-line. J. Diff. Eq. 258 (2015), 3107-3160.

[H1] J. Holmer, The initial-boundary-value problem for the Korteweg-de Vries equation. Comm. Partial Differential Equations 31 (2006), 1151-1190.

[H2] J. Holmer, The initial-boundary-value problem for the $1 D$ nonlinear Schrödinger equation on the half-line. Diff. Int. Eq. 18 (2005), 647-668.

[K1] T. Kato, On nonlinear Schrödinger equations. Ann. Inst. H. Poincaré Phys. Theor. 46 (1987), 113-129.

[K2] T. Kato, On nonlinear Schrödinger equations. II. $H^{s}$-solutions and unconditional well-posedness. J. Anal. Math. 67 (1995), 281-306.

[KPV1] C. Kenig, G. Ponce and L. Vega, Oscillatory integrals and regularity of dispersive equations. Indiana U. Math. J. 40 (1991), 33-69.

[KPV2] C. Kenig, G. Ponce and L. Vega, On the ill-posedness of some canonical dispersive equations. Duke Math. J. 106 (2001), 617-633.

[KPV3] C. Kenig, G. Ponce and L. Vega, Small solutions to nonlinear Schrödinger equations. Ann. Inst. H. Poincaré Anal. Non Linéaire 10 (1993), 255-288.

[KTV] R. Killip, T. Tao and M. Visan, The cubic nonlinear Schrödinger equation in two dimensions with radial data. J. Eur. Math. Soc. 11 (2009), 1203-1258.

$[\mathrm{KV}] \quad$ R. Killip and M. Visan, Global well-posedness and scattering for the defocusing quintic NLS in three dimensions. Anal. PDE 5 (2012), 855-885.

[KSS] K. Kirkpatrick, B. Schlein and G. Staffilani, Derivation of the two-dimensional nonlinear Schrödinger equation from many body quantum dynamics. Amer. J. Math. 133 (2011), 91-130.

[LPSS] M. Landman, G. Papanicolaou, C. Sulem and P.-L. Sulem, Rate of blowup for solutions of the nonlinear Schrödinger equation at critical dimension. Phys. Rev. A 38 (1988), 3837.

[LF1] J. Lenells and A. Fokas, The unified method: II. NLS on the half-line with t-periodic boundary conditions. J. Phys. A: Math. Theor. 45 (2012), 195202. 
[LF2] J. Lenells and A. Fokas, The unified method: III. Nonlinearizable problems on the interval. J. Phys. A: Math. Theor. 45 (2012), 195203.

[LP] F. Linares and G. Ponce, Introduction to nonlinear dispersive equations, Universitext, Springer, 2009.

[LM] J. Lions and E. Magenes, Non-homogeneous boundary value problems and applications, Vol. 1, SpringerVerlag, 1972.

[MF] D. Mantzavinos and A. Fokas, The unified method for the heat equation: II. Non-separable boundary conditions in two dimensions. Euro. J. Appl. Math. 26 (2015), 887-916.

[M] F. Merle, Determination of blow-up solutions with minimal mass for nonlinear Schrödinger equations with critical power. Duke Math. J. 69 (1993), 427-454.

[MR] F. Merle and P. Raphael, On universality of blow-up profile for $L^{2}$ critical nonlinear Schrödinger equation. Invent. Math. 156 (2004), 565-672.

[P] D. Peregrine, Water waves, nonlinear Schrödinger equations and their solutions. J. Austral. Math. Soc. Ser. B 25 (1983), 16-43.

[PS] L. Pitaevskii and S. Stringari, Bose-Einstein condensation, Clarendon Press, 2003.

[RSZ] Y. Ran, S. Sun and B.Y. Zhang, Nonhomogeneous initial boundary value problems of 2D nonlinear Schrödinger equations in a half-plane. arXiv: 1609.05418v2 (2017).

[S] R. Strichartz, A guide to distribution theory and Fourier transforms, CRC, 1994.

[SB] W. Strauss and C. Bu, An inhomogeneous boundary value problem for nonlinear Schrödinger equations. J. Diff. Eq. 173 (2001), 79-91.

[SS] C. Sulem and P.-L. Sulem, The nonlinear Schrödinger equation: self-focusing and wave collapse, Springer, 1999.

[Ta] V. Talanov, Self-focusing of electromagnetic waves in nonlinear media. Radiophysics 8 (1964), $254-257$.

[TM1] M. Tsutsumi, On smooth solutions to the initial-boundary value problem for the nonlinear Schrödinger equation in two space dimensions. Nonlinear Anal. 13 (1989), 1051-1056.

[TM2] M. Tsutsumi, On global solutions to the initial-boundary value problem for the nonlinear Schrödinger equations in exterior domains. Comm. Partial Differential Equations 16 (1991), 885-907.

[T1] Y. Tsutsumi, Global solutions of the nonlinear Schrödinger equation in exterior domains. Comm. Partial Differential Equations 8 (1983), 1337-1374.

[T2] Y. Tsutsumi, $L^{2}$-solutions for nonlinear Schrödinger equations and nonlinear groups. Funkcialaj Ekvacioj 30 (1987), 115-125.

[V] M. Visan, The defocusing energy-critical nonlinear Schrödinger equation in higher dimensions. Duke Math. J. 138 (2007), 281-374.

[WW] J. Weiland and H. Wiljelmsson, Coherent nonlinear interaction of waves in plasmas, Pergamon Press, 1977.

[W] M. Weinstein, Nonlinear Schrödinger equations and sharp interpolation estimates. Comm. Math. Phys. 87 (1982), 567-576.

[Z] V. Zakharov, Stability of periodic waves of finite amplitude on the surface of a deep fluid. J. Appl. Mech. Tech. Phys. 9 (1968), 190-194.

[ZS] V. Zakharov and A. Shabat, Exact theory of two-dimensional self-focusing and one-dimensional selfmodulation of waves in nonlinear media. Sov. Phys. JETP 34 (1972), 63-69.

A. Alexandrou Himonas

Department of Mathematics

University of Notre Dame

Notre Dame, IN 46556

E-mail: himonas.1@nd.edu
Dionyssios Mantzavinos

Department of Mathematics

University of Kansas

Lawrence, KS 66045

E-mail: mantzavinos@ku.edu

\section{Appendix: Solution of the Forced Linear IBVP via Fokas' Unified Transform}

We provide a concise derivation of the unified transform formula (1.7) for the forced linear Schrödinger IBVP (1.6) under the assumption of smooth initial and boundary values. For a more detailed derivation, we refer the reader to [F3], where formula (1.7) was first obtained. 
Let $\widetilde{u}$ satisfy the adjoint of the linear Schrödinger equation, i.e. $i \widetilde{u}_{t}-\widetilde{u}_{x_{1} x_{1}}-\widetilde{u}_{x_{2} x_{2}}=0$. Multiplying this equation by the solution $u$ of the forced linear Schrödinger equation (1.6a), and adding to it equation (1.6a) multiplied by $\tilde{u}$, we arrive at the divergence form

$$
i(\widetilde{u} u)_{t}+\left(\widetilde{u} u_{x_{1}}-\widetilde{u}_{x_{1}} u\right)_{x_{1}}+\left(\widetilde{u} u_{x_{2}}-\widetilde{u}_{x_{2}} u\right)_{x_{2}}=\widetilde{u} f .
$$

Setting $\widetilde{u}\left(x_{1}, x_{2}, t\right)=e^{-i k_{1} x_{1}-i k_{2} x_{2}+i\left(k_{1}^{2}+k_{2}^{2}\right) t}, k_{1}, k_{2} \in \mathbb{C}$ and integrating with respect to $x_{1}$ and $x_{2}$ while assuming that $u \rightarrow 0$ as $\left|x_{1}\right|,\left|x_{2}\right| \rightarrow \infty$, we obtain

$$
i\left(e^{i\left(k_{1}^{2}+k_{2}^{2}\right) t} \widehat{u}\left(k_{1}, k_{2}, t\right)\right)_{t}=-e^{i\left(k_{1}^{2}+k_{2}^{2}\right) t}\left[\widehat{u}_{x_{2}}^{x_{1}}\left(k_{1}, 0, t\right)+i k_{2} \widehat{u}^{x_{1}}\left(k_{1}, 0, t\right)\right]+e^{i\left(k_{1}^{2}+k_{2}^{2}\right) t} \widehat{f}\left(k_{1}, k_{2}, t\right),
$$

where $\widehat{u}$ and $\widehat{f}$ are the half-plane Fourier transforms of $u$ and $f$ defined by (1.8) while $\widehat{u}^{x_{1}}$ denotes the Fourier transform of $u$ with respect to $x_{1}$ defined as in (1.3).

Note that $\widehat{u}$ and $\widehat{f}$ are analytic as functions of $k_{2}$ in $\mathbb{C}^{-}=\left\{k_{2} \in \mathbb{C}: \operatorname{Im}\left(k_{2}\right)<0\right\}$ as a consequence of the following Paley-Wiener theorem.

Theorem A.1 ([S], Theorem 7.2.4). For any $\phi \in L^{2}(0, \infty)$, the half-line Fourier transform

$$
\widehat{\phi}(k)=\int_{x=0}^{\infty} e^{-i k x} \phi(x) d x
$$

is analytic in $\mathbb{C}^{-}=\{k \in \mathbb{C}: \operatorname{Im}(k)<0\}$.

Integrating (A.1) with respect to $t$ gives rise to the so-called global relation

$$
\begin{aligned}
e^{i\left(k_{1}^{2}+k_{2}^{2}\right) t} \widehat{u}\left(k_{1}, k_{2}, t\right)= & \widehat{u}_{0}\left(k_{1}, k_{2}\right)+i \widetilde{g}_{1}\left(k_{1}, k_{1}^{2}+k_{2}^{2}, t\right)-k_{2} \widetilde{g}_{0}\left(k_{1}, k_{1}^{2}+k_{2}^{2}, t\right) \\
& -i \int_{t^{\prime}=0}^{t} e^{i\left(k_{1}^{2}+k_{2}^{2}\right) t^{\prime}} \widehat{f}\left(k_{1}, k_{2}, t^{\prime}\right) d t^{\prime}, \quad\left(k_{1}, k_{2}\right) \in \mathbb{R} \times \overline{\mathbb{C}^{-}},
\end{aligned}
$$

where for $g_{j}\left(x_{1}, t\right)=\partial_{x_{2}}^{j} u\left(x_{1}, 0, t\right), j=0,1$, we define

$$
\widetilde{g}_{j}\left(k_{1}, k_{1}^{2}+k_{2}^{2}, t\right)=\int_{t^{\prime}=0}^{t} e^{i\left(k_{1}^{2}+k_{2}^{2}\right) t^{\prime}} \widehat{g}_{j}^{x_{1}}\left(k_{1}, t^{\prime}\right) d t^{\prime}, \quad j=0,1 .
$$

Inverting (A.2) by means of the usual inverse Fourier transform, we obtain

$$
\begin{aligned}
& u\left(x_{1}, x_{2}, t\right)=\frac{1}{(2 \pi)^{2}} \int_{k_{1} \in \mathbb{R}} \int_{k_{2} \in \mathbb{R}} e^{i k_{1} x_{1}+i k_{2} x_{2}-i\left(k_{1}^{2}+k_{2}^{2}\right) t} \widehat{u}_{0}\left(k_{1}, k_{2}\right) d k_{2} d k_{1} \\
& -\frac{i}{(2 \pi)^{2}} \int_{k_{1} \in \mathbb{R}} \int_{k_{2} \in \mathbb{R}} e^{i k_{1} x_{1}+i k_{2} x_{2}-i\left(k_{1}^{2}+k_{2}^{2}\right) t} \int_{t^{\prime}=0}^{t} e^{i\left(k_{1}^{2}+k_{2}^{2}\right) t^{\prime}} \widehat{f}\left(k_{1}, k_{2}, t^{\prime}\right) d t^{\prime} d k_{2} d k_{1} \\
& +\frac{1}{(2 \pi)^{2}} \int_{k_{1} \in \mathbb{R}} \int_{k_{2} \in \mathbb{R}} e^{i k_{1} x_{1}+i k_{2} x_{2}-i\left(k_{1}^{2}+k_{2}^{2}\right) t}\left[i \widetilde{g}_{1}\left(k_{1}, k_{1}^{2}+k_{2}^{2}, t\right)-k_{2} \widetilde{g}_{0}\left(k_{1}, k_{1}^{2}+k_{2}^{2}, t\right)\right] d k_{2} d k_{1} .
\end{aligned}
$$

The representation (A.3) is not an explicit solution formula for the forced linear IBVP (1.6) because it involves the unknown Neumann boundary value $u_{x}(0, t)$ through the transform $\widetilde{g}_{1}$. However, it turns out that $\widetilde{g}_{1}$ can be eliminated from (A.3) in favor of known quantities. In particular, note that since $x_{2} \geqslant 0$ and $t \geqslant t^{\prime}$ the exponential $e^{i k_{2} x_{2}-i k_{2}^{2}\left(t-t^{\prime}\right)}$ is bounded for all $k_{2} \in \overline{\mathbb{C}^{+} \backslash D}$, where $D$ here denotes the first quadrant of the complex $k_{2}$-plane. Thus, exploiting the analyticity of $\widetilde{g}_{j}$ for all $k_{2} \in \mathbb{C}$ (which follows via a Paley-Wiener theorem similar to Theorem A.1) we apply Cauchy's theorem in the second quadrant of the complex $k_{2}$-plane to write the $k_{2}$-integral in the last term of (A.3) as

$$
\int_{k_{2} \in \partial D} e^{i k_{2} x_{2}-i k_{2}^{2} t}\left[\tilde{g}_{1}\left(k_{1}, k_{1}^{2}+k_{2}^{2}, t\right)-k_{2} \widetilde{g}_{0}\left(k_{1}, k_{1}^{2}+k_{2}^{2}, t\right)\right] d k_{2}-\lim _{\rho \rightarrow \infty} I\left(\rho, k_{1}, x_{2}, t\right),
$$


where $\partial D$ is the positively oriented boundary of $D$ depicted in Figure 1.1 and for the quarter circle $\gamma_{\rho}^{+}=\left\{\rho e^{i \theta}, \frac{\pi}{2} \leqslant \theta \leqslant \pi\right\}$ shown in Figure A.1 we define

$$
I\left(\rho, k_{1}, x_{2}, t\right)=\int_{k_{2} \in \gamma_{\rho}^{+}} e^{i k_{2} x_{2}-i k_{2}^{2} t}\left[i \widetilde{g}_{1}\left(k_{1}, k_{1}^{2}+k_{2}^{2}, t\right)-k_{2} \widetilde{g}_{0}\left(k_{1}, k_{1}^{2}+k_{2}^{2}, t\right)\right] d k_{2}
$$

Integrating by parts with respect to $t^{\prime}$ in the definitions of $\widetilde{g}_{0}$ and $\widetilde{g}_{1}$, we obtain

$$
(\mathrm{A} .4) \lesssim \frac{C_{g_{0}, g_{1}}\left(k_{1}\right)}{x_{2}} \cdot \frac{\rho\left(1-e^{-\rho x_{2}}\right)}{\left|\rho^{2}-k_{1}^{2}\right|}
$$

where, recalling that we work under the assumption of smooth boundary values,

$$
C_{g_{0}, g_{1}}\left(k_{1}\right)=\left\|{\widehat{g_{1}}}^{x_{1}}\left(k_{1}\right)\right\|_{L_{t}^{\infty}(0, T)}+\left\|{\widehat{g_{0}}}^{x_{1}}\left(k_{1}\right)\right\|_{L_{t}^{\infty}(0, T)}+\left\|\partial_{t}{\widehat{g_{1}}}^{x_{1}}\left(k_{1}\right)\right\|_{L_{t}^{\infty}(0, T)}+\left\|\partial_{t} \widehat{g}_{0}^{x_{1}}\left(k_{1}\right)\right\|_{L_{t}^{\infty}(0, T)}<\infty
$$

Hence, the integral (A.4) vanishes in the limit $\rho \rightarrow \infty$ and, in turn, the integral representation (A.3) reads

$$
\begin{aligned}
& u\left(x_{1}, x_{2}, t\right)=\frac{1}{(2 \pi)^{2}} \int_{k_{1} \in \mathbb{R}} \int_{k_{2} \in \mathbb{R}} e^{i k_{1} x_{1}+i k_{2} x_{2}-i\left(k_{1}^{2}+k_{2}^{2}\right) t} \widehat{u}_{0}\left(k_{1}, k_{2}\right) d k_{2} d k_{1} \\
& -\frac{i}{(2 \pi)^{2}} \int_{k_{1} \in \mathbb{R}} \int_{k_{2} \in \mathbb{R}} e^{i k_{1} x_{1}+i k_{2} x_{2}-i\left(k_{1}^{2}+k_{2}^{2}\right) t} \int_{t^{\prime}=0}^{t} e^{i\left(k_{1}^{2}+k_{2}^{2}\right) t^{\prime}} \widehat{f}\left(k_{1}, k_{2}, t^{\prime}\right) d t^{\prime} d k_{2} d k_{1} \\
& +\frac{1}{(2 \pi)^{2}} \int_{k_{1} \in \mathbb{R}} \int_{k_{2} \in \partial D} e^{i k_{1} x_{1}+i k_{2} x_{2}-i\left(k_{1}^{2}+k_{2}^{2}\right) t}\left[i \widetilde{g}_{1}\left(k_{1}, k_{1}^{2}+k_{2}^{2}, t\right)-k_{2} \widetilde{g}_{0}\left(k_{1}, k_{1}^{2}+k_{2}^{2}, t\right)\right] d k_{2} d k_{1} .
\end{aligned}
$$

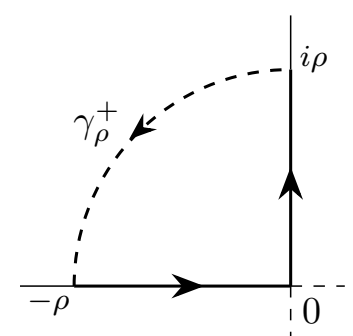

Figure A.1. Cauchy's theorem in the second quadrant of the complex $k_{2}$-plane.

Next, note that under the transformation $k_{2} \mapsto-k_{2}$ the global relation (A.2) yields the identity

$$
\begin{aligned}
e^{i\left(k_{1}^{2}+k_{2}^{2}\right) t} \widehat{u}\left(k_{1},-k_{2}, t\right)= & \widehat{u}_{0}\left(k_{1},-k_{2}\right)+i \widetilde{g}_{1}\left(k_{1}, k_{1}^{2}+k_{2}^{2}, t\right)+k_{2} \widetilde{g}_{0}\left(k_{1}, k_{1}^{2}+k_{2}^{2}, t\right) \\
& -i \int_{t^{\prime}=0}^{t} e^{i\left(k_{1}^{2}+k_{2}^{2}\right) t^{\prime}} \widehat{f}\left(k_{1},-k_{2}, t^{\prime}\right) d t^{\prime}, \quad\left(k_{1}, k_{2}\right) \in \mathbb{R} \times \overline{\mathbb{C}^{+}} .
\end{aligned}
$$

Thanks to the fact that

$$
\int_{k_{2} \in \partial D} e^{i k_{2} x_{2}} \widehat{u}\left(k_{1},-k_{2}, t\right) d k_{2}=0
$$


due to the analyticity and exponential decay of the integrand inside $D$, we are able to solve identity (A.6) for $\widetilde{g}_{1}$ and substitute the resulting expression in (A.5) to obtain the explicit solution formula

$$
\begin{aligned}
u\left(x_{1}, x_{2}, t\right)= & \frac{1}{(2 \pi)^{2}} \int_{k_{1} \in \mathbb{R}} \int_{k_{2} \in \mathbb{R}} e^{i k_{1} x_{1}+i k_{2} x_{2}-i\left(k_{1}^{2}+k_{2}^{2}\right) t} \widehat{u}_{0}\left(k_{1}, k_{2}\right) d k_{2} d k_{1} \\
& -\frac{1}{(2 \pi)^{2}} \int_{k_{1} \in \mathbb{R}} \int_{k_{2} \in \partial D} e^{i k_{1} x_{1}+i k_{2} x_{2}-i\left(k_{1}^{2}+k_{2}^{2}\right) t} \widehat{u}_{0}\left(k_{1},-k_{2}\right) d k_{2} d k_{1} \\
& -\frac{i}{(2 \pi)^{2}} \int_{k_{1} \in \mathbb{R}} \int_{k_{2} \in \mathbb{R}} e^{i k_{1} x_{1}+i k_{2} x_{2}-i\left(k_{1}^{2}+k_{2}^{2}\right) t} \int_{t^{\prime}=0}^{t} e^{i\left(k_{1}^{2}+k_{2}^{2}\right) t^{\prime}} \widehat{f}\left(k_{1}, k_{2}, t^{\prime}\right) d t^{\prime} d k_{2} d k_{1} \\
& +\frac{i}{(2 \pi)^{2}} \int_{k_{1} \in \mathbb{R}} \int_{k_{2} \in \partial D} e^{i k_{1} x_{1}+i k_{2} x_{2}-i\left(k_{1}^{2}+k_{2}^{2}\right) t} \int_{t^{\prime}=0}^{t} e^{i\left(k_{1}^{2}+k_{2}^{2}\right) t^{\prime}} \widehat{f}\left(k_{1},-k_{2}, t^{\prime}\right) d t^{\prime} d k_{2} d k_{1} \\
& +\frac{1}{(2 \pi)^{2}} \int_{k_{1} \in \mathbb{R}} \int_{k_{2} \in \partial D} e^{i k_{1} x_{1}+i k_{2} x_{2}-i\left(k_{1}^{2}+k_{2}^{2}\right) t} 2 k_{2} \widetilde{g}_{0}\left(k_{1}, k_{1}^{2}+k_{2}^{2}, t\right) d k_{2} d k_{1} .
\end{aligned}
$$

Finally, exploiting once again analyticity and exponential decay in $D$, we infer that

$$
\int_{k_{2} \in \partial D} e^{i k_{2} x_{2}-i k_{2}^{2} t} \int_{t^{\prime}=t}^{T} e^{i\left(k_{1}^{2}+k_{2}^{2}\right) t^{\prime}} \widehat{g}_{0}^{x_{1}}\left(k_{1}, t^{\prime}\right) d t^{\prime} d k_{2}=0
$$

and hence the solution formula (A.7) can be written in the equivalent form (1.7), which is the one convenient for deriving linear estimates. 